\title{
Efficient and Accurate Evaluation Methods for Concordance Measures via Functional Tensor Characterizations of Copulas
}

\author{
Antonio Dalessandro ${ }^{1}$. Gareth W. Peters ${ }^{2}$ (D)
}

Received: 31 July 2018 / Revised: 24 October 2019 / Accepted: 25 October 2019 /

Published online: 5 December 2019

(C) The Author(s) 2019

\begin{abstract}
There is now an increasingly large number of proposed concordance measures available to capture, measure and quantify different notions of dependence in stochastic processes. However, evaluation of concordance measures to quantify such types of dependence for different copula models can be challenging. In this work, we propose a class of new methods that involves a highly accurate and computationally efficient procedure to evaluate concordance measures for a given copula, applicable even when sampling from the copula is not easily achieved. In addition, this then allows us to reconstruct maps of concordance measures locally in all regions of the state space for any range of copula parameters. We believe this technique will be a valuable tool for practitioners to understand better the behaviour of copula models and associated concordance measures expressed in terms of these copula models.
\end{abstract}

Keywords Concordance measures - Copula functions - Copula infinitesimal generators · Martingale problem - Multidimensional semimartingales decomposition approximations . Semimartingales decomposition · Tensor algebra

Mathematics Subject Classification (2010) 47N30 - 60B15 • 46N30 - 62G32 - 62H86

In Dalessandro and Peters (2017) and Dalessandro and Peters (2016) the authors developed a novel class of functional copula representations for dependence that can be viewed as a reinterpretation of Sklars' well known copula representation theorem of multivariate dependence. This is achieved in a manner that allows one to characterize any copula dependence

Antonio Dalessandro

info@antonio-dalessandro.com

Gareth W. Peters

g.peters@hw.ac.uk

1 Department of Statistical Science, University College London, London, UK

2 Department of Actuarial Mathematics and Statistics, Heriot-Watt University, Edinburgh, UK 
function via a unique map to a generalized Gaussian copula function. This unique mapping is obtained through the quantification of local dependence properties of the reference copula model over a discretized representation. Furthermore, the authors also demonstrated that such a representation is proven to be exact as the discretization interval of the target copulas support diminishes, with known convergence rate as studied in detail in Dalessandro and Peters (2017). In this manuscript we extend and develop this representation for the purpose of calculating and evaluation concordance measures, which can be written as functionals of copula models. These can include concepts such as multivariate upper negative (positive) dependence, lower negative (positive) dependence, negative (positive) dependence; multivariate negative (positive) quadrant dependence; multivariate association, co-monotinicity, stochastic ordering; regression dependence negative (positive) and extreme dependence, asymptotic tail dependence and intermediate tail dependence; as well as other concepts such as directional dependence, see Joe $(1990,1997)$ and Nelsen (2002). Measuring the dependence between random variables has long been of interest to statisticians and practitioners alike. A history of the development of dependency measures can be found in Mari and Kotz (2001). One should realise that in general, the dependence structure between two random variables can only be captured in full by their joint probability distribution, and thus any scalar quantity extracted from this structure must be viewed as a representation of some feature of the dependence.

Scarsini (1984) gives the following intuitive definition of dependence:

Dependence is a matter of association between $X$ and $Y$ along any measurable function, i.e. the more $X$ and $Y$ tend to cluster around the graph of a function, either $y=f(x)$ or $x=g(y)$, the more they are dependent.

The choice of dependence measure is influenced by the type of dependence one seeks to study, such as lower left quadrant, upper right quadrant, etc. However, in nontrivial multivariate distributions, it is not possible to capture all of the possible combinations of dependence patterns within a single dependence measure. For this reason there is now an increasingly large number of proposed concordance measures available to capture, measure and quantify different notions of dependence in stochastic processes. The study of such measures of dependence began in pairwise constructions in the works of Cambanis et al. (1976), Tchen (1980) and Yanagimoto and Okamoto (1969). Where they demonstrated that an ordering on discrete bivariate distributions, which formalized the notion of concordance, was shown to be equivalent to stochastic ordering of distribution functions with identical marginals. These notions were then generalised in multivariate settings by for instance Joe (1990) and Scarsini (1984) with the latter introducing a formal axiomatic representation of multivariate concordance measures given in Proposition 1. A detailed overview of these concepts is provided in Cruz et al. (2015).

Definition 1 Consider the following basic definitions for permutation and symmetry, used throughout.

- Symmetries: a symmetry of $[0,1]^{d}$ is a one-to-one, onto map $\phi:[0,1]^{d} \mapsto[0,1]^{d}$ of form $\phi\left(x_{1}, \ldots, x_{d}\right)=\left(u_{1}, \ldots, u_{d}\right)$ where for each $i$ one has $u_{i}=x_{k_{i}}$ or $1-x_{k_{i}}$ and where $\left(k_{1}, \ldots, k_{d}\right)$ is a permutation of $(1, \ldots, n)$;

- Permutation: the map $\phi$ is a permutation if for each $i$ one has $u_{i}=x_{k_{i}}$;

- Reflection: the map $\phi$ is a reflection if for each $i$ one has $u_{i}=x_{i}$ or $u_{i}=1-x_{i}$. 
- Elementary reflections: an elementary reflection of the $i$-th component, denoted $\sigma_{i}$ is given by

$$
\sigma_{i}\left(x_{1}, \ldots, x_{d}\right)=\left(x_{1}, \ldots, x_{i-1}, 1-x_{i}, x_{i+1}, \ldots, x_{d}\right)
$$

- Symmetry Length: the length of a symmetry is denoted by $|\phi|$ and corresponds to the number elementary reflections required to obtain it.

We can now proceed to provide the general form of axioms for specification of a multivariate concordance measure as detailed in Scarsini (1984).

Proposition 1 (Multivariate Concordance Measures) A general concordance measures $\kappa$ is a function attaching to all d-tuples of continuous r.v.'s $\left(X_{1}, X_{2}, \ldots, X_{d}\right)$ defined on a common probability space, when $d \geq 2$, a real number $\kappa\left(X_{1}, X_{2}, \ldots, X_{d}\right)$ satisfying:

- Normalization: $\kappa\left(X_{1}, X_{2}, \ldots, X_{d}\right)=1$ if each $X_{i}$ is a.s. an increasing function of every other $X_{j}$ and $\kappa\left(X_{1}, X_{2}, \ldots, X_{d}\right)=0$ if $X_{1}, \ldots, X_{d}$ are independent;

- Monotonicity: If $X_{1}, \ldots, X_{d}$ is less concordent than $Y_{1}, \ldots, Y_{d}$ then $\left(X_{1}, X_{2}, \ldots, X_{d}\right)<\kappa\left(Y_{1}, Y_{2}, \ldots, Y_{d}\right)$;

- Continuity: If $F_{k}$ is the joint distribution of $\left(X_{k 1}, \ldots, X_{k d}\right)$ and $F$ the distribution of $\left(X_{1}, \ldots, X_{d}\right)$ and one has convergence in the sequence $F_{k} \rightarrow F$ as $k \rightarrow \infty$, then $\kappa\left(X_{k 1}, \ldots, X_{k d}\right) \rightarrow \kappa\left(X_{1}, \ldots, X_{d}\right)$;

- Permutation Invariance: If $\left(i_{1}, \ldots, i_{d}\right)$ is a permutation of $(1, \ldots, d)$ then $\kappa\left(X_{i_{1}}, \ldots, X_{i_{d}}\right)=\kappa\left(X_{1}, \ldots, X_{n}\right)$;

- Duality: $\kappa\left(-X_{1}, \ldots,-X_{n}\right)=\kappa\left(X_{1}, \ldots, X_{n}\right)$;

- Reflection Symmetry: $\sum_{\epsilon_{1}, \ldots, \epsilon_{d}= \pm 1} \kappa\left(\epsilon_{1} X_{1}, \ldots, \epsilon_{d} X_{d}\right)=0$ where the sum is over $2^{d}$ vectors of the form $\left(\epsilon_{1} X_{1}, \ldots, \epsilon_{d} X_{d}\right)$ with $\epsilon_{i} \in\{-1,1\}$;

- Transition: There exists a sequence $\left\{r_{d}\right\}$ for $d \geq 2$ such that every $d$-tuple of continuous r.v.'s $\left(X_{1}, \ldots, X_{d}\right)$ satisfies

$$
r_{d-1} \kappa\left(X_{2}, \ldots, X_{d}\right)=\kappa\left(X_{1}, \ldots, X_{d}\right)+\kappa\left(-X_{1}, X_{2}, \ldots, X_{d}\right)
$$

In addition, there is an increasing number of copula models aimed at modelling dependence and in many cases there is no clear and intuitive correspondence between the magnitude of a copula's parameters and the dependence structure they create. Furthermore the form of concordance and the strength of that concordance measure in different dimensions as a function of the value of the parameters of the copula model. We view the work created in this manuscript as a useful class of representations and tools that can help study such features in non-trivial copula models.

In Taylor (2007) they provided a representation of the axioms of a concordance measure from Scarsini (1984) early work, rewritten explicitly in terms of copula models. This provides a link between these measures of dependence and the copula model, however a good understanding of the strength or significance of a concordance measure as a function of the copula model parameters is not well understood and difficult to study at present. The main reason for this is that often the evaluation of these concordance measures for different copula models can be very challenging and typically does not admit simple closed form solutions except in some special well known cases. There are many copula families in which a framework such as the one we propose in this manuscript will facilitate efficient and computationally accurate methods to gain an understanding of the relationships between different notions of concordance and the copula models parameters. 
Therefore, the aim of this paper is to develop a class of numerical approximations that practitioners can utilise to study locally in the state space of the multivariate random vector two important features: firstly the effects of parametric specifications of dependence in the form of different copula models and their local induced concordance structures; and secondly the role of each parameter in the copula model specifications in varying the concordance measure strength locally in the state space. This should be achievable for any copula model and any desired concordance measure. To achieve these goals we extend the class of methods developed in Dalessandro and Peters (2017) and Dalessandro and Peters (2016) by developing a highly accurate and computationally efficient procedure to evaluate concordance measures for a given copula.

In Dalessandro and Peters (2017) a theoretical framework for tensor representation is developed and theoretical convergence properties are proven along with computational rates of convergence and complexity. Then in Dalessandro and Peters (2016) they propose a generic model for approximating any target copula function which will allow us to develop a clear and efficient characterization of their properties locally in any desired region of the state space (support of the target copula model). This characterization admits a accurate reconstruction of a given copula that we will demonstrate in this paper allows one to then utilise to accurately calculate a wide range of concordance measures of dependence both locally and globally in the support of the given target copula model. We note that we present all required quantities from these two papers in this manuscript to make this paper stand alone.

We recall that a copula is simply a multivariate probability distribution for which the marginal probability distribution of each variable is uniform (Nelsen 1999). Copulas are often used in high-dimensional statistical applications as they allow one to separate out the modelling and estimation of the distribution of dependent random variables by estimating first the marginals and then capturing the dependence structure through estimation of a copula function.

Outline of Contributions In Section 1 we will recall briefly the core results of the approach developed in Dalessandro and Peters (2016) to construct an approximation of a copula distribution. This involves a decomposition of the representation of the copula according to a tensor algebraic method of construction based on continuous Markov chains approximation of generalized diffusions. The link then to the copula approximation is that the generalized diffusion is constructed to admit a density at time $t$ which satisfies the desired copula or dependence structure to be studied. In this manner, we can construct through approximations of the generalised diffusion with a copula structure between the marginals of the process, any desired static copula model approximation that will be of relevance to statistical modelling in understanding relationships between copula parameters and concordance structures present.

In this paper we extend these results to utilise the copula model decompositions to evaluate efficiently and locally in the state space of the random vector a wide range of multivariate concordance measures of dependence. To achieve this in Section 2 we present the relationship between concordance measures and copula models and then introduce our tensor algebraic approximations for a range of multivariate concordance measures for different copula families. This allows us to study these concordance measures as a function of the copula parameters to better understand how the copula parameters induce dependence and what type of dependence is present for these copula models. This is far from trivial to understand in the grouped, generalized and skewed Student's t-copula cases and the Archimax 
cases. We finish with illustrations of approximations of concordance measures for multivariate rank correlations, directional dependences, intermediate tail dependence and asymptotic tail dependence measures.

\section{Approximation Scheme for a Copula via a Tensor Decomposition}

In this section we will explain how to perform the representation of a target copula distribution through a tensor approximation of generalized diffusions, which will form the basis for all the constructions derived for concordance measures. The methodology is based on results derived in detail in Dalessandro and Peters (2017) and then developed for copula modelling in Dalessandro and Peters (2016) and which we briefly recall in Appendix A.

We denote by $\mathbf{x}:=\left(x_{1}, \ldots, x_{d}\right) \in \mathbb{R}^{d}$ a d-dimensional vector, and inequalities $\mathbf{x} \leq \mathbf{z}$ are intended componentwise, i.e., $x_{i} \leq z_{i}$ for all $i=1,2, \ldots, d$. Let $\mathbf{X}_{t}^{(n)}$ a d-dimensional Markov chain with $d \geq 2$, specifically with $X_{t}^{\left(n_{1}\right)}, X_{t}^{\left(n_{2}\right)}, \ldots, X_{t}^{\left(n_{d}\right)}$ marginal chains, each with support $\mathcal{X}^{k}=\left\{x_{1}^{(k)}, x_{2}^{(k)}, \ldots, x_{n_{k}}^{(k)}\right\} \subset \mathbb{R}$, where $n_{1}, n_{2}, \ldots$ denotes the number of corresponding chains' states. Therefore

$$
\mathbf{X}_{t}^{(n)}: \mathcal{X} \rightarrow \mathbb{R}^{d}
$$

with $\mathcal{X}=\bigotimes_{i=1}^{d} \mathcal{X}^{\mathbf{i}} \in \mathbb{R}^{d}$ denoting the d-dimensional tensor space and $n=n_{1} \cdots n_{d}$.

We will use the equivalent notation $\mathbf{X}_{t}^{\left(n_{1}, n_{2}, \ldots, n_{d}\right)}$ for the Markov chain $\mathbf{X}_{t}^{(n)}$. We denote the Markov chain joint distribution function by

$$
F_{t}^{(n)}(\mathbf{x})=P_{t}^{(n)}\left(\mathbf{X}_{t}^{(n)} \leq \mathbf{x}\right), \quad \mathbf{x} \in \mathcal{X} \subset \mathbb{R}^{d},
$$

with marginal distribution functions

$$
F_{t}^{\left(n_{k}\right)}\left(x_{k}\right)=P_{t}^{\left(n_{k}\right)}\left(X_{t}^{\left(n_{k}\right)} \leq x_{k}\right), x_{k} \in \mathbb{R}, k=1, \ldots, d .
$$

We specify the approximated copula function as the joint distribution function of the Markov chain

$$
\mathbf{U}_{t}^{(n)}=F_{t}^{(n)}\left(\mathbf{X}_{t}^{(n)}\right)
$$

with marginal chains $U_{t}^{\left(n_{k}\right)}=F_{t}^{\left(n_{k}\right)}\left(X_{t}^{\left(n_{k}\right)}\right)$, for $k=1, \ldots, d$, each with support $\mathbf{U}^{k}=$ $F_{t}^{\left(n_{k}\right)}\left(\mathbf{X}^{k}\right)=\left\{u_{1}^{(k)}, u_{2}^{(k)}, \ldots, u_{n_{k}}^{(k)}\right\} \subset[0,1]$, where $F_{t}^{\left(n_{k}\right)}$ denotes the application of the distribution function of the Markov chain $X_{t}^{\left(n_{k}\right)}$ to each point, for all $k=1, \ldots, d$.

We denote the approximated copula function by $C^{(n)}: \mathbf{U} \rightarrow[0,1]$, where $\mathbf{U}=$ $\bigotimes_{i=1}^{d} \mathbf{U}^{i} \subset[0,1]^{d}$ is the discretized unit hypercube, such that

$$
F_{t}^{(n)}(\mathbf{x})=C^{(n)}\left(F_{t}^{\left(n_{1}\right)}\left(x_{1}\right), \ldots, F_{t}^{\left(n_{d}\right)}\left(x_{d}\right)\right), \text { for all } \mathbf{x} \in \mathcal{X} \in \mathbb{R}^{d} .
$$

Moreover if $\mathbf{u}:=\left(u_{1}, \ldots, u_{d}\right) \in \mathbf{U} \subset[0,1]^{d}$ is a d-dimensional vector in the unit hypercube, we have that

$$
C^{(n)}(\mathbf{u})=F_{t}^{(n)}\left(F_{t}^{-1,\left(n_{1}\right)}\left(u_{1}\right), \ldots, F_{t}^{-1,\left(n_{d}\right)}\left(u_{d}\right)\right) .
$$

We denote by $c^{(n)}\left(\mathbf{u} ; \theta_{c}\right)$ and $C^{(n)}\left(\mathbf{u}, \theta_{c}\right)$ the copula density and the copula distribution function respectively, for all vectors $\mathbf{u} \in \mathbf{U} \subseteq[0,1]^{d}$ with copula parameters' set $\theta_{c}$.

Then according to Dalessandro and Peters (2016), we calculate the copula as

$$
c^{(n)}(\mathbf{u})=\frac{P_{t}^{(n)}(\mathbf{x} ;\{\theta, \rho(\mathbf{u})\})}{P_{t}^{(n)}(\mathbf{x} ;\{\theta, 0\})}, \text { for } \mathbf{u} \in \mathbf{U}, \text { for } \mathbf{x}=F^{-1,(n)}(\mathbf{u})
$$


with $t=1$, and $\{\theta, \rho(\mathbf{u})\}$ denoting the set of the Markov chain parameters with $\rho(\mathbf{u}): \mathbf{U} \rightarrow$ $[-1,1]$ the local correlation function. Note that every coordinate vector $\mathbf{u}$ in the discretized hypercube $\mathbf{U}$ may exhibit a specific mapped local correlation, $\rho(\mathbf{u}) \in[-1,1]$.

In particular the parameters' set $\theta_{c}$ of $C^{(n)}(\mathbf{u})=C^{(n)}\left(\mathbf{u}, \theta_{c}\right)$, the copula distribution function approximated on the unit hypercube $\mathbf{U}$ in Eq. 1.6, are linked to the Markov chain distribution function by the relation

$$
C^{(n)}\left(\mathbf{u} ; \theta_{c}\right)=F_{1}^{(n)}(\mathbf{u} ;\{\theta, \rho(\mathbf{u})\}), \text { for all } \mathbf{u} .
$$

Given a target copula distribution function $C:=C_{\text {target }}$, and $C_{\text {target }}^{(n)}\left(\mathbf{u} ; \theta_{c}\right)$, its discrete evaluation on the unit hypercube points $\mathbf{u} \in \mathbf{U}$, the equivalent representation in a tensor space is obtained by locally solving the following problem

$$
\min _{\rho(\mathbf{u})}\left\|C_{\text {target }}^{(n)}\left(\mathbf{u} ; \theta_{c}\right)-F_{1}^{(n)}(\mathbf{x} ;\{\theta, \rho(\mathbf{u})\})\right\|_{2} \text { for all } \mathbf{u} .
$$

Equation 1.9 is of key importance in our approach because it allows us to link the often complex and not so intuitive target copula parameters set $\theta_{c}$ to the local Gaussian correlation parameter set $\rho(\mathbf{u})$. In this way the copula parametric dependence structure is explained and displayed through an equivalent representation by means of local Gaussian correlation coefficients $\rho(\mathbf{u})$.

A practical way to solve (1.9) is to compute the local likelihood with respect to each local Gaussian correlation parameter

$$
\min _{\rho(\mathbf{u})}\left\|\log \left(C_{\text {target }}^{(n)}\left(\mathbf{u} ; \theta_{c}\right)-C^{(n)}(\mathbf{u} ;\{\theta, \rho(\mathbf{u})\})\right)\right\|_{2}, \quad \text { for all } \mathbf{u} \in \mathbf{U} .
$$

where $C_{\text {target }}$ can be any target copula function. The copula distribution function $C^{(n)}=$ $C^{(n)}(\mathbf{u} ;\{\theta, \rho(\mathbf{u})\})$ is evaluated in all points $\mathbf{u}=\left(u_{1}, \ldots, u_{d}\right) \in \mathbf{U}$ and its value is of a Gaussian copula function with parameter $\rho(\mathbf{u})$ (Figs. 1 and 2).
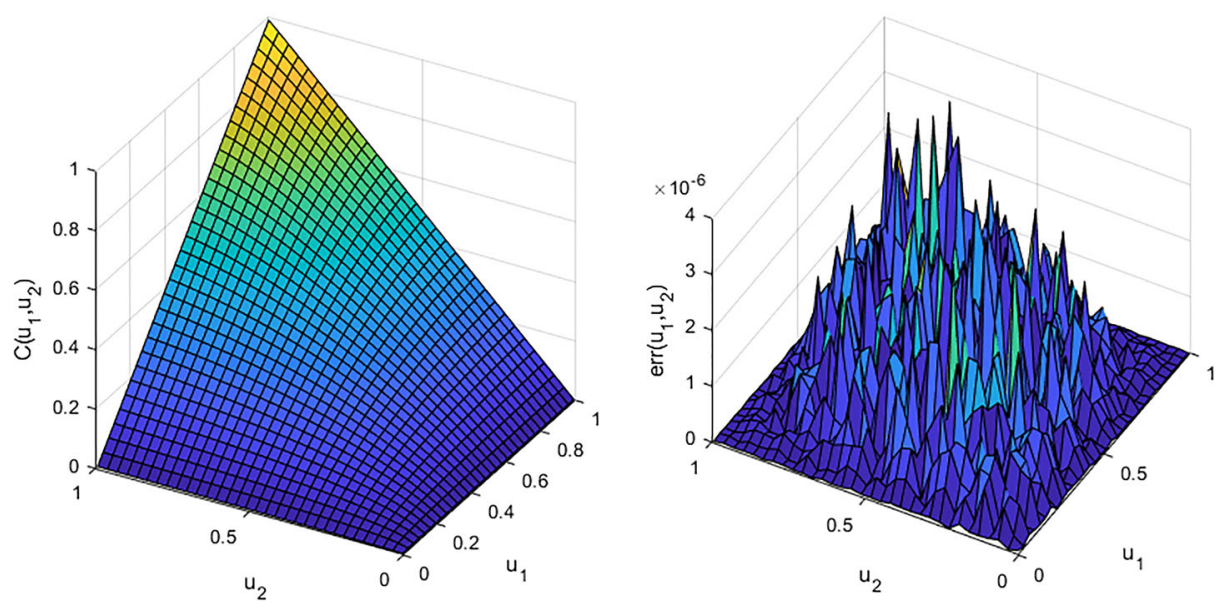

Fig. 1 The left plot reports the copula distribution function $C_{\text {target }}^{(n)}$ belonging to Student-t copula, evaluated on the unit square $\mathbf{U}:=\bigotimes_{i=1}^{2} \mathbf{U}^{i} \in[0,1]^{2}$ and with parameters $\theta_{c}=\{\rho=0.5, v=5\}$. The number of states used in this example is $n_{1}=n_{2}=35$. This represents the discretized target copula distribution, or the copula distribution we seek to approximate. The right end side plot reports the error from solving (1.10) across the unit square $\mathbf{U}$, namely $\min _{\rho(\mathbf{u})}\left\|C_{\text {target }}^{(n)}\left(\mathbf{u} ; \theta_{c}\right)-C^{(n)}(\mathbf{u} ;\{\theta, \rho(\mathbf{u})\})\right\|_{2}$ for all $\mathbf{u} \in \mathbf{U}$ 

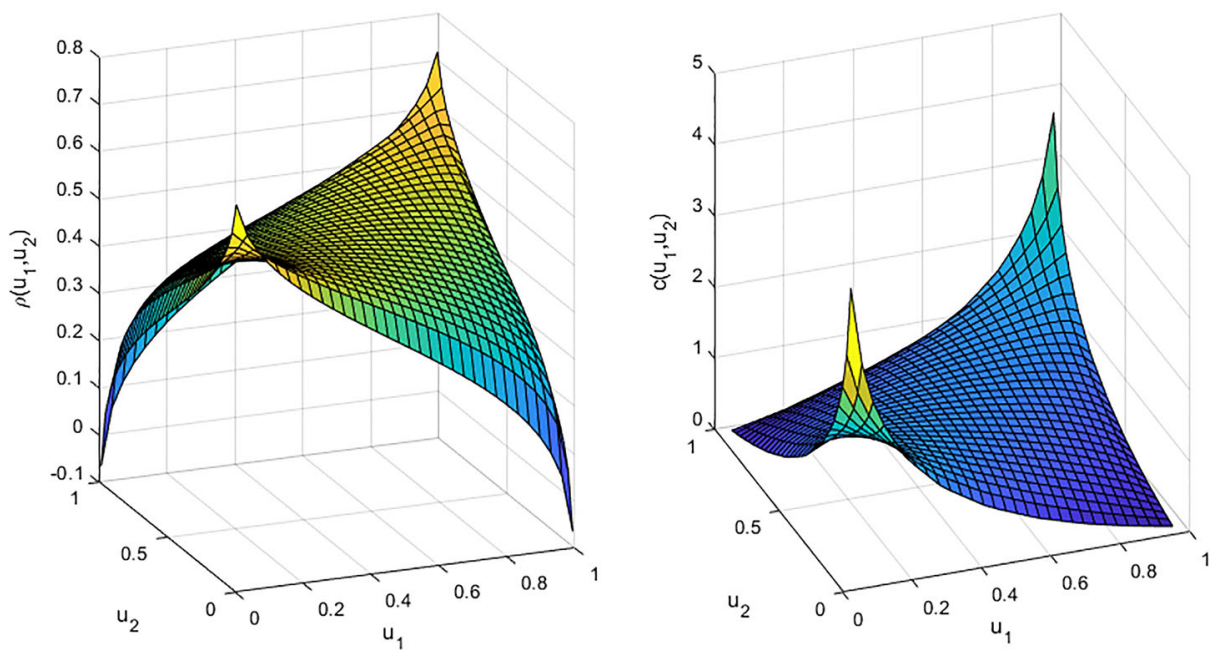

Fig. 2 The plot on the left end side reports $\rho(\mathbf{u})$ for all $\mathbf{u} \in \mathbf{U}$ from solving (1.10) which represent the local Gaussian correlations equivalent to the Student t-copula parameters $\theta_{c}=\{\rho=0.5, v=5\}$. This plot is the visualization of the dependence structure of the target Student t-copula by means of local Gaussian correlation parameters $\rho(\mathbf{u})$ for all $\mathbf{u} \in \mathbf{U}$. Each point coordinate $\mathbf{u}=\left(u_{1}, u_{2}\right)$ on the discretized copula support $\mathbf{U}$ is uniquely characterized by a Gaussian correlation value $\rho\left(u_{1}, u_{2}\right)$ which gives the magnitude of the dependency between the marginals. The plot on the right side displays the calculated Student-t copula density $c(\mathbf{u},\{\theta, \rho(\mathbf{u})\})$ function of the Gaussian local correlations $\rho(\mathbf{u})$ for all $\mathbf{u} \in \mathbf{U}$

\subsection{Properties of the Approximated Local Gaussian Copula $c^{(n)}$}

In this section we present some important properties of the approximated local Gaussian copula function $c^{(n)}$ which makes our proposed methodology to mimic approximated target copulas extremely appealing when dealing with calculations involving copulas and specifically the concordance measures.

In particular:

1. We give another explanation to the way we construct the copula approximation. We prove that the approximating function $c^{(n)}$ is interpretable the approximating density or Radon-Nikodym derivative of the approximated measure $P^{(n)}$ with respect to $P^{(n) \perp}$, where the notation for the approximated density $P^{(n)}$ and approximated uncorrelated density $P^{(n) \perp}$ is as described in Appendix A, specifically see Eq. A.9.

2. The local minimizer in Eq. 1.10 always admits a solution.

Remark 1.1 (Orthogonality of the tensor basis) By construction the approximated local copula density function $c^{(n)}$ is defined on a orthogonal basis, which is the basis resulting from the tensor product of the constituent operators. In fact each operator matrix used in its calculation is orthogonal by construction, see Appendix A. Furthermore we construct the copula over the support $\mathcal{X}$ which may be represented as union of disjoint subsets $\left\{B_{j}\right\}$, i.e. $\mathcal{X}=\bigotimes_{i=1}^{d} \mathcal{X}^{i}=\bigcup_{j=1}^{n} B_{j}$, where each set $B_{j}$ as coordinate vector point $\mathbf{x}$ in the space $\mathbf{X}$. If we then set $B_{0}=\left\{\mathbf{x}=\left(x_{0}^{(1)}, \ldots, x_{0}^{(d)}\right)\right\}, B_{1}=\left\{\mathbf{x}=\left(x_{1}^{(1)}, \ldots, x_{1}^{(d)}\right)\right\}, \ldots, B_{n}=$ $\left\{\mathbf{x}=\left(x_{n_{1}}^{(1)}, \ldots, x_{n_{d}}^{(d)}\right)\right\}$, therefore creating a countable and ordered sequence of sets spanning 
the whole discretized support $\mathcal{X}$ then it is straightforward to obtain the copula distribution functions as the cumulative sum over all the sets, i.e.

$$
C^{(n)}\left(B_{z}\right)=\sum_{j \leq z} c^{(n)}\left(B_{j}\right)
$$

In order to prove the above properties we first need to remind some basic definitions of the density and conditional distribution of a copula.

Definition 2 Let $F_{1}, \ldots, F_{d}$ be continuous marginal distributions. Let $\mathrm{C}$ be a copula distribution

$$
C(\mathbf{u})=F\left(F_{1}^{-1}\left(u_{1}\right), \ldots, F_{d}^{-1}\left(u_{d}\right)\right)
$$

When the copula distribution $\mathrm{C}$ and the joint distribution $\mathrm{F}$ are both differentiable, the joint density function $P$ satisfies

$$
c(\mathbf{u})=\frac{P\left(F_{1}^{-1}\left(u_{1}\right), \ldots, F_{d}^{-1}\left(u_{d}\right)\right)}{\left.P_{1}\left(F_{1}^{-1}\left(u_{1}\right)\right) \cdots P_{d} F_{d}^{-1}\left(u_{d}\right)\right)}=c\left(F_{1}\left(x_{1}\right), \ldots, F_{d}\left(x_{d}\right)\right)
$$

with $c$ denoting the copula density which is linked to the distribution $\mathrm{C}$ by

$$
c(\mathbf{u})=\frac{\partial^{d}}{\partial u_{1} \ldots d u_{d}} C(\mathbf{u}) .
$$

Therefore the copula density is the ratio of the joint density $P(\mathbf{x}), \mathbf{x} \in \mathbb{R}^{d}$ and the density under independence which is equivalent to the product of the marginal density function $P_{1}\left(x_{1}\right) \cdots P_{d}\left(x_{d}\right)$. It is therefore possible to interpret the copula as the adjustment that we need to make to convert the independence pdf into the joint pdf.

However this adjustment can also have another interpretation: a Radon-Nikodym (R-N) derivative. In our specific case it will be a R-N derivative for the discrete measure $P^{(n)}$ constructed through the local Gausian CTMC approximation of Dalessandro and Peters (2017) which is summarized in Appendix A.

If in Eq. 1.13 we set $P(\mathbf{u})=P\left(F_{1}^{-1}\left(u_{1}\right), \ldots, F_{d}^{-1}\left(u_{d}\right)\right)$ and $P^{\perp}(\mathbf{u})=$ $\left.P_{1}\left(F_{1}^{-1}\left(u_{1}\right)\right) \cdots P_{d} F_{d}^{-1}\left(u_{d}\right)\right)$ for convenience of notation then

$$
P(\mathbf{u})=c(\mathbf{u}) P^{\perp}(\mathbf{u}) .
$$

Let us give some more insights about the interpretation of Eq. 1.15 in a more general mathematical setting and the way the copula $c^{(n)}(\mathbf{u})$ is constructed in our proposed model.

For this purpose $X=(X, \mathcal{F})$ is a measurable space, and we shall refer to the elements of $\mathcal{F}$ simply as measurable sets omitting the $\sigma$-algebra $\mathcal{F}$. All subsets of $X$ and all functions on $X$ appearing below are measurable unless otherwise indicated.

Definition 3 (Radon-Nikodym Theorem) Let $\mu$ and $v$ be two continuous measures on $X$, with $v$ is $\sigma$-finite and $\mu$ absolutely continuous with respect to $v$. Then there exists a positive function such that

$$
\mu(B)=\int_{B} f d v
$$

for all subsets $B$ of $\mathrm{X}$. The function $f$ is unique in the sense that if $\mu(B)=\int_{B} g d v$ for all $B$ then $f=g$. The function $\mathrm{f}$ is called the Radon-Nikodym derivative or density of $\mu$ with respect to $\nu$ and is denoted by $\frac{d \mu}{d \nu}$. 
Note that a necessary and sufficient condition that the Radon-Nikodym derivative $f$ be integrable is that $\mu$ is $\sigma$-finite. If we restrict the Radon-Nikodym derivative $f$ to the space $(X, \mathcal{G})$ with $\mathcal{G} \in \mathcal{F}$ a $\sigma$-subalgebra, the Radon-Nikodym derivative is

$$
\left.\frac{d \mu}{d \nu}\right|_{\mathcal{G}}=\frac{1}{P(\mathcal{G})} \int_{\mathcal{G}} f d \mu=\mathbb{E}[f \mid \mathcal{G}] .
$$

We also remind the law of total expectation which plays an import role in the way we construct the approximated copula density.

Definition 4 If $X$ is a random variable whose expected value is $\mathbb{E}[X]$, and if $\left\{B_{j}\right\}, i=$ $1,2, \ldots$ is a finite or countable partition of the sample space, then

$$
\mathbb{E}[X]=\sum_{j} \mathbb{E}\left[X \mid B_{j}\right] P\left(B_{j}\right)
$$

We are now in a position to explain what is the relationship between a R-N derivative $\lambda$ and all its restrictions $g_{j}=\mathbb{E}\left[\lambda \mid B_{j}\right]$ over disjoint sets $\left\{B_{j}\right\}, i=1,2, \ldots$, and we do this in the discrete settings of the copula $c^{(n)}$.

Proposition 2 (Construction of the Approximated Copula $c^{n}$ ) The copula $c^{(n)}: \mathbf{U} \rightarrow$ $[0, \infty)$ in Eq. 1.15 is the approximating density or $R-N$ derivative of the approximated measure $P^{(n)}$ with respect to $P^{(n) \perp}$. Furthermore $c(n)$ it is the results of the averaging of $R-N$ $g_{j}=\mathbb{E}\left[\lambda \mid B_{j}\right]$ which are the restriction of $c(n)$ on disjoint set $B_{j}$ spanning $\mathcal{X}=\bigotimes_{i} \mathcal{X}^{i}$.

Proof From Eq. 1.15 we have that the copula density $c^{(n)}$ is interpretable as R-N derivative of the approximated correlated measure $P^{(n)}$ with respect to the absolutely continuous measure $P^{(n) \perp}$ for all sets in $\mathbf{U}$. Equivalently for the approximated copula $c^{(n)}$ we have that $P^{(n)}(A)=\mathbb{E}\left[1_{A} c^{(n)}\right]$ for $A \in \mathcal{X}$. We then observe that in our framework the density support $\mathcal{X}=\bigcup_{j=1}^{n} B_{j}$ can be represented as the union of countable disjoint sets $B_{j}$. Therefore we can apply the law of total expectation to the discrete R-N derivative and obtain:

$$
\mathbb{E}\left[1_{A} c^{(n)}\right]=\mathbb{E}\left[\sum_{j} \frac{\mathbb{E}\left[c^{(n)} 1_{B_{j}}\right]}{P\left(B_{j}\right)} 1_{B_{j}}\right]=\sum_{j} \mathbb{E}\left[c^{(n)} 1_{B_{j}}\right]=g_{j} \mathbb{E}\left[1_{B_{j}}\right]
$$

being the variable $g_{j}$ is measurable with respect to $B_{j}$.

In our proposed framework we approximate the first two moments of each conditional variable $g_{j}=\mathbb{E}\left[\lambda \mid B_{j}\right]$ with the first two moments of a conditional normal variable. Details of the local construction of each approximated conditional variable are in Appendix A.

Using Eq. 1.19 we can calculate a local conditional value, which is Gaussian in our case, matching the local target copula value, and this is done by means of the local minimizer of Eq. 1.10. The existence of the local minimizer is explained by the above properties of the R$\mathrm{N}$ derivative. The fact that in this setting always exists a bounded $\mathrm{R}-\mathrm{N}$ derivative linking the two densities, and the approximation of the restricted R-N derivative to a conditional Gaussian density, guarantees the existence of a local Gaussian copula value and its corresponding correlation $\rho(\mathbf{u})$.

Theorem 1 [Fréchet-Hoeffding bounds, see Embrechts et al. 2001] If C is a d-dimesional copula, then for every $\mathbf{u} \in[0,1]^{d}$, the Fréchet-Hoeffding inequality is

$$
W^{d}(\mathbf{u}) \leq C(\mathbf{u}) \leq M^{d}(\mathbf{u})
$$


where the functions $W^{d}(\mathbf{u})$ and $M^{d}(\mathbf{u})$ defined on $[0,1]^{d}$ are as following:

$$
\begin{aligned}
& W^{d}(\mathbf{u})=\max \left(u_{1}+\ldots+u_{d}-d+1,0\right) \\
& M^{d}(\mathbf{u})=\min \left(u_{1}, \ldots, u_{d}\right) .
\end{aligned}
$$

In particular the function $M^{d}$ is a d-dimensional copula for all $d$, however $W^{d}$ is not a copula for $d>3$.

Proposition 3 (Existence of the Minimizer of Eq. 1.10) Given a target d-dimensional copula distribution $C$ with parameters set $\theta_{c}$, which we denote by $C_{\text {target }}\left(\mathbf{u} ; \theta_{c}\right)$ and a generalized local Gaussian copula $C^{(n)}$ defined on the discretized hypecube $\mathbf{U}$ we have that the local minimizer

$$
\min _{\rho(\mathbf{u})}\left\|C_{\text {target }}^{(n)}\left(\mathbf{u} ; \theta_{c}\right)-C^{(n)}(\mathbf{u} ;\{\theta, \rho(\mathbf{u})\})\right\|_{2}
$$

always exists for all $\mathbf{u} \in \mathbf{U}$.

Proof We observe that by the Fréchet-Hoeffding bounds Theorem 1 we have that for all $\mathbf{u} \in \mathbf{U}$

$$
W^{d}(\mathbf{u})=C^{(n)}(\mathbf{u} ;\{\theta,-1\}) \leq C^{(n)}(\mathbf{u} ;\{\theta, \rho(\mathbf{u})\}) \leq C^{(n)}(\mathbf{u} ;\{\theta, 1\})=M^{d}(\mathbf{u})
$$

However we have that such bounds are valid for any copula and in particular

$$
W^{d}(\mathbf{u}) \leq C_{\text {target }}\left(\mathbf{u} ; \theta_{c}\right) \leq M^{d}(\mathbf{u})
$$

Therefore for any given coordinate point $\mathbf{u} \in \mathbf{U}$ it is always possible to find a correlation value $\rho(\mathbf{u}) \in[-1,1]$ which minimizes the local minimizer of Eq. 1.21.

\subsection{Direct Measure of Directional Dependence}

In this section we show how our framework gives immediate measure of directional dependence. At this purpose we recall some basic definitions.

Definition 5 [Directional Dependence] The pair $\left(U_{1}, U_{2}\right)$ is directionally dependent in joint behaviour if

$$
\mathbb{E}\left[U_{1} \mid U_{2}=w\right] \neq \mathbb{E}\left[U_{2} \mid U_{1}=w\right]
$$

Directional dependence can be expressed in terms of regression using a copula distribution function, see Sungur (2005).

Definition 6 (Directional Dependence through regression, see Sungur 2005) Let $\left(U_{1}, U_{2}\right)$ denote a pair of random variables whose marginal distributions have uniform distribution on $[0,1]$ and the joint distribution is a copula function $C\left(u_{1}, u_{2}\right)$. Let $C_{u_{2}}\left(u_{1}\right)$ denote the conditional distribution of $U_{1}$ given $U_{2}=\bar{u}$

$$
C_{u_{2}}\left(u_{1}\right)=P\left(U_{1} \leq u_{1} \mid U_{2}=\bar{u}\right)=\frac{\partial C\left(u_{1}, u_{2}\right)}{\partial u_{2}} .
$$

The copula regression function of $U_{2}$ on $U_{1}$ is the conditional expectation of $U_{1}$ given $U_{2}=\bar{u}$, which can be expressed by the copula as

$$
r_{U_{1} \mid U_{2}}(\bar{u}):=\mathbb{E}\left[U_{1} \mid U_{2}=\bar{u}\right]=1-\int_{0}^{1} C_{u_{2}}\left(u_{1}\right) d u_{1} .
$$


The directional dependence from $U_{2}$ to $U_{1}$ is defined by using the copula regression function on $U_{1}$ as

$$
\rho_{U_{2} \rightarrow U_{1}}^{2}=\frac{\operatorname{Var}\left(r_{U_{1} \mid U_{2}}(\bar{u})\right)}{\operatorname{Var}\left(U_{1}\right)}=\frac{\mathbb{E}\left[\left(r_{U_{1} \mid U_{2}}(\bar{u})-0.5\right)^{2}\right]}{1 / 12}=12 \mathbb{E}\left[\left(r_{U_{1} \mid U_{2}}(\bar{u})\right)^{2}\right]-3
$$

which can be interpreted as the proportion of total variance of $U_{1}$ that has been explained by the copula regression function $r_{U_{1} \mid U_{2}}(\bar{u})$.

We can immediately calculate (1.26) in our framework which can be rewritten in terms of approximated local Gaussian copula as

$$
r_{U_{1} \mid U_{2}}^{(n)}(\bar{u})=1-\sum_{i} C_{u^{(2)}}^{(n)}\left(u_{i}^{1}\right) \Delta u^{(1)}
$$

where

$$
C_{u_{j}^{(2)}}^{(n)}\left(u_{i}^{(1)}\right)=\frac{1}{\Delta u_{j}^{(2)}}\left(C^{(n)}\left(u_{i}^{(1)}, u_{j}^{(2)}+\Delta u_{j}^{(2)}\right)-C^{(n)}\left(u_{i}^{(1)}, u_{j}^{(2)}\right)\right)
$$

with $\mathbf{U}^{k}=\left\{u_{1}^{(k)}, u_{2}^{(k)}, \ldots, u_{n_{k}}^{(k)}\right\}$ the discretized support of the uniform marginals as introduced above in Eq. 1.4, and $\Delta u_{i}^{(k)}=u_{i+1}^{(k)}-u_{i}^{(k)}$. Note that each support grid $U^{k}$ is not uniformly spaced.

\section{Understanding Relationships Between Copula Parameter(s) and Concordance Measures}

In this section we discuss the relationship between concordance measures of dependence and the copula representations presented in the previous section. In particular we illustrate how to utilise such copula representations to develop a clearer understanding of the relationship between a copula parameter and different measures of concordance which can be generally applied to any form of dependence model captured by a copula. The choice of dependence measure or concordance measure is influenced by the type of dependence one seeks to study, such as: multivariate upper negative (positive) dependence, lower negative (positive) dependence and negative (positive) dependence; multivariate negative and positive quadrant dependence; multivariate association, co-monotonicity and stochastic ordering; positive and negative regression dependence; and extreme dependence, tail dependence and intermediate tail dependence. We start by discussing recent relationships between general notions of concordance measures between random variables and their characterization through copula parametric models. We recall first a general definition of a concordance measure for a random vector.

Definition 7 (Concordance Measures for Random Vectors) A pair of random variables are concordant if 'large' values of one tend to be associated with 'large' values of the other and 'small' values of one with 'small' values of the other. Analogous definitions of discordance are available in reverse directions. 
There are numerous ways of mathematically trying to quantify this statement, so consequently, many measures of concordance are available. Such a definition, offers the intuition behind the need for measuring and quantifying concordance. However, from a statistical perspective a more formal understanding of concordance measures was mathematically stated with regard to copula models in the recent works of Taylor (2007). This selection of axioms that are desirable for a concordance measure to satisfy are based on the copula characterization of the earlier framework originally developed by Scarsini (1984) and presented in Proposition 1. In Taylor (2007) the following axioms for general concordance measures $\kappa$ specified generically via copula $C$ are detailed below in Proposition 4.

Proposition 4 (Multivariate Concordance Measures via Copula) Consider a sequence of maps $\kappa_{d}: \operatorname{Cop}(d) \mapsto \mathbb{R}$ and a sequence of numbers $\left\{r_{d}\right\}$, such that if $A, B, C$ and $C_{m}$ are $d$-copulas and $n \geq 2$ then:

- Normalization: $\kappa\left(M^{d}\right)=1$ and $\kappa\left(\Pi^{d}\right)=0$.

- Monotonicity: If $A<_{s t} B$ and $\bar{A} \leq_{s t} \bar{B}$ then $\kappa_{d}(A) \leq \kappa_{d}(B)$

- Continuity: If $C_{m} \rightarrow C$, then $\kappa_{d}\left(C_{m}\right) \rightarrow \kappa_{d}(C)$ as $m \rightarrow \infty$.

- Permutation Invariance: If $\left(i_{1}, \ldots, i_{d}\right)$ is a permutation of $(1, \ldots, d)$ then $\kappa\left(c\left(u_{i_{1}}, \ldots, u_{i_{d}}\right)\right)=\kappa\left(c\left(u_{1}, \ldots, u_{d}\right)\right.$.

- Duality: $\kappa_{d}\left(c\left(1-u_{1}, \ldots, 1-u_{d}\right)=\kappa_{d}\left(c\left(u_{1}, \ldots, u_{d}\right)\right)\right.$

- Reflection Symmetry: $\sum_{\Psi \in \mathcal{R}_{d}} \kappa_{d}\left(C^{\Psi}\right)=0$, where $\Psi$ is a reflection, $\Psi \in \mathcal{R}_{d}$ is an element of the subgroup of reflections in the group of symmetries under composition $\mathcal{S}\left([0,1]^{d}\right)$.

- Transition:

$$
r_{n} \kappa_{d}(C)=\kappa_{n+1}(E)+\kappa_{n+1}\left(E\left(1-u_{1}, u_{2}, \ldots, u_{d}\right)\right)
$$

whenever $E$ is an $(d+1)$-copula s.t. $C\left(u_{1}, \ldots, u_{d}\right)=E\left(1, u_{1}, \ldots, u_{d}\right)$.

Although we know these general relationships between the axioms that describe concordance and dependence structures in general and we now know them in general terms as specified by a statistical model in the form of a copula, it still remains a real challenge to evaluate and understand these relationships with regard to model parameters for a given copula model. We aim to address this challenge using the representations of copula developed previously in the paper.

We note that one can also state the following theorem regarding the properties of concordance measures that satisfy these axioms, see details in Taylor (2007).

Theorem 2 (Properties of Concordance Measures Satisfying Proposition 4)

Consider the d-copula that is permutation symmetric ie. $C^{\zeta}=C$ for all permuations $\zeta$ of $[0,1]^{d}$. Then for all measures of concordance $\kappa$ and for all symmetries $\Psi$ and $\zeta$ of $[0,1]^{d}$ one has

$$
\kappa_{d}\left(C^{\Psi}\right)=\kappa_{d}\left(C^{\zeta}\right)
$$

whenever $|\Psi|=|\zeta|$ or $|\Psi|+|\zeta|=d$

Recall: symmetry length $|\cdot|$ corresponds to the number elementary reflections required to obtain it. 
Corollary 1 For all $d \geq 2$ and for all symmetries $\Psi$ and $\zeta$ of $[0,1]^{d}$ such that $|\Psi|=|\zeta|$ or $|\Psi|+|\zeta|=d$ one has

$$
\kappa_{d}\left(M^{\Psi}\right)=\kappa_{d}\left(M^{\zeta}\right)
$$

where $M$ is the d-Frechet-Hoffding Upper Bound copula under permutation.

With regard to copula models there are numerous measures one can consider that satisfy all or some subset of such axioms. Typically, the evaluation of these concordance measures and the understanding of the relationship between these concordance measures and the copula parameter(s) is non-trivial and changes as a function of both dimension and copula parameter(s) value highly non-linearly. In this section we first discuss some important examples of concordance measures and then we detail how the framework developed previously may be utilise to provide a natural framework for evaluation of such measures, thus providing the necessary understanding of the relationships between the copula family, copula parameter(s) and the behavior of the concordance measure.

\subsection{Functional Copula Mapping for Approximation of General Multivariate Concordance Measures}

Arguably the most widely known and utilised measure of dependence, Pearson's Product Moment Correlation Coefficient, was developed by Karl Pearson, see Pearson (1896), building on Sir Francis Galton's approach using the median and semi-interquartile range, see Galton (1894). Pearson's correlation coefficient is a measure of how well the two random variables can be described by a linear function. The other popular measures often used in practice when considering notions of pairwise dependence are Spearman's $\rho$ (Spearman 1904), Kendall's $\tau$ (Kendall 1938) and Blomqvist's $\beta$ (Blomqvist 1950). In the case of Spearman's $\rho$ it is a measure that assesses how well the dependence between two random variables can be described by a monotonic function. As such it is equivalent to the Pearson's correlation coefficient between the ranked variables. In addition, one can consider Spearman's rho and Kendall's $\tau$ each as a simple scalar measures of dependence that depend on the copula of two random variables but not on their marginal distributions. As noted in Fredricks and Nelsen (2007), one can consider Spearman's $\rho$ as forming a measure of average quadrant dependence, while Kendall's $\tau$ is a measure of average likelihood ratio dependence. In the case of Blomqvist's $\beta$ the generalized form is discussed in any dimension in Joe (1990), Dolati and Úbeda-Flores (2006), and Nelsen (2002) where it is shown to be expressed directly in terms of a copula distribution function. However, unlike Kendalls $\tau$ and Spearmans $\rho$ which are functionals integrated against the copula distribution, the Blomqvists $\beta$ is simple to evaluate given the copula distribution function. It simply requires the evaluation of the copula distribution at a point $\bar{u}=[1 / 2, \ldots, 1 / 2]$. We therefore focus below on the evaluation of functionals with regard to the constructed copula and demonstrate how to evaluate these efficiently.

First we observe the following results for Spearman's and Kendall's Rank correlations, both can be specified directly in terms of a copula distribution in both the bivariate and multivariate settings. We begin with the specification of multivariate Spearman rank. 


\subsubsection{Multivariate Spearman Rank Correlation Approximations}

Definition 8 (Spearman's Rank Correlation via Copula) The bivariate Spearman's Rank Correlation can be expressed explicitly via the bivariate copula $C$ according to

$$
\rho=12 \int_{[0,1]} \int_{[0,1]} u_{1} u_{2} d C\left(u_{1}, u_{2}\right)-3 .
$$

In addition, a general multivariate extension of Spearman's Rank Correlation is developed for $d$-dimensional loss random vectors and given below, see details in Nelsen (2002).

Definition 9 (Multivariate Spearman's Rho via Copula) Consider the $d$-copula given by $C$, then Spearman's Rho concordance measure of dependence is given by

$$
\rho(C)=h(d)\left[2^{d} \int_{[0,1]^{d}} C(u) d u-1\right]=h(d)\left[2^{d} \int_{[0,1]^{d}} \Pi(u) d C(u)-1\right]
$$

where $h(d)=\frac{d+1}{2^{d}-(d+1)}$ is the normalizing factor derived such that the maximum correlation is equal to 1 .

Definition 10 (Multivariate Generalized Spearman's Rho via Copula) Consider the $d$ copula given by $C$ and the permuted copula $C^{\sigma}$, then the generalized Spearman's Rho concordance measure of dependence is given according to

$$
\rho_{d}(C)=\alpha_{d}\left(\int_{[0,1]^{d}}\left(C+C^{\sigma}\right) d \Pi^{d}-\frac{1}{2^{d-1}}\right)
$$

where one has $\alpha_{d}=\frac{(d+1) 2^{d-1}}{2^{d}-(d+1)}$ and $\Pi^{d}$ is the $d$-Independence Copula.

From this definition of the generalized Spearman's Rho rank correlation, we can also define the local as well as the constrained versions of the state-space rank correlation.

Definition 11 (Local and Constrained Multivariate Generalized Spearman's Rho via Copula) Consider the $d$-copula given by $C$ and the permuted copula $C^{\sigma}$, then the local generalized Spearman's Rho concordance measure of dependence for vectors $\mathbf{u}$ localized in some sub-space $\mathbf{u} \in \Omega \subset[0,1]^{d}$ is given according to

$$
\rho_{d}(C ; \Omega)=\alpha_{d}\left(\int_{\Omega}\left(C+C^{\sigma}\right) d \Pi^{d}-\frac{1}{2^{d-1}}\right)
$$

where one has $\alpha_{d}=\frac{(d+1) 2^{d-1}}{2^{d}-(d+1)}$ and $\Pi^{d}$ is the $d$-Independence Copula. The constrained version of the Spearman's Rho is then given for vectors $\mathbf{u}$ constrained in some sub-space $\mathbf{u} \in \Omega \subset[0,1]^{d}$ according to

$$
\tilde{\rho}_{d}(C ; \Omega)=\alpha_{d}\left(\int_{\Omega}\left(\widetilde{C}+\widetilde{C}^{\sigma}\right) d \widetilde{\Pi}^{d}-\frac{1}{2^{d-1}}\right)
$$

where one has $\alpha_{d}=\frac{(d+1) 2^{d-1}}{2^{d}-(d+1)}$ and $\widetilde{\Pi}^{d}$ is the $d$-Independence Copula. Where the $\widetilde{C}$ notation refers to the copula distribution given after renormalization by the truncation according to

$$
\widetilde{C}(B ; \Omega)=\int_{B} \frac{c(u)}{C(\Omega)} d u
$$


for all sets $B \in \Omega$.

An example where such a correlation measure is of interest arises when one considers intermediate and extremal dependence measures, such as the following illustrative bivariate examples where given a pair of uniform variable such that $\left(U_{1}, U_{2}\right) \sim C\left(u_{1}, u_{2}\right)$ and one wishes to evaluate the truncated correlation or restricted correlations given for instance by

$$
\begin{aligned}
& \mathbb{E}\left[U_{1} U_{2} \mid U_{1}>u_{1}, U_{2}>u_{2}\right]=\int_{[0,1]} \int_{[0,1]} u_{1} u_{2} d C\left(u_{1} u_{2} \mid U_{1}>u_{1}, U_{2}>u_{2}\right) \\
& \mathbb{E}\left[U_{1} U_{2} \mid U_{1} \leq u_{1}, U_{2}>u_{2}\right]=\int_{[0,1]}^{0} \int_{[0,1]}^{0} u_{1} u_{2} d C\left(u_{1} u_{2} \mid U_{1} \leq u_{1}, U_{2}>u_{2}\right) .
\end{aligned}
$$

The important observation is that in general it can be very difficult to evaluate such quantities even using Monte Carlo procedures, as sampling the conditional or constrained copulas is a great challenge in many families of copula. In the the following we specify an efficient approximation based on the general functional copula mapping developed previously.

Proposition 5 (Approximating Local and Constrained Multivariate Generalized Spearman's Rho via Functional Copula Mappings) Consider the $d$-copula given by $C$ which is discretely approximated by the functional copula mapping given by $C^{(n)}(\mathbf{u})$ for all vectors $\mathbf{u} \in \mathbf{U}^{k}, k=1, \ldots, d$, and $C^{(n) \sigma}$ represents the functional copula mapping of the permuted copula $C$ obtained after permuting the base mimicking local Gaussian copula mapping $C^{(n)}$. Then one has the functional approximations to the localized generalized Spearman's Rho concordance measure of dependence in some sub-space $\Omega \subseteq \mathbf{U} \subset[0,1]^{d}$ is given according to

$$
\begin{aligned}
& \rho_{d}^{(n)}\left(C^{(n)} ; \Omega\right) \\
& =\alpha_{d} \sum_{\mathbf{u} \in \Omega}\left[C^{(n)}(\mathbf{u})+C^{(n) \sigma}(\mathbf{u})\right] \prod_{i=1}^{d}\left(\mathbf{u}-\mathbf{u}^{\prime}\right)-\frac{\alpha_{d}}{2^{d-1}}
\end{aligned}
$$

where one has $\alpha_{d}=\frac{(d+1) 2^{d-1}}{2^{d}-(d+1)}$ and $\mathbf{u}^{\prime} \in \Omega$ is the neighbour of $\mathbf{u}$.

The constrained version of the Spearman's Rho is then given for vectors $\mathbf{u} \in \bar{\Omega} \subseteq \mathbf{U}$, therefore for vectors in a constrained some sub-space $\bar{\Omega} \subset[0,1]^{d}$ according to

$$
\begin{aligned}
& \tilde{\rho}_{d}^{(n)}(C ; \bar{\Omega}) \\
& =\alpha_{d} \sum_{\mathbf{u} \in \bar{\Omega}}\left[\widetilde{C}^{(n)}(\mathbf{u})+\widetilde{C}^{(n) \sigma}(\mathbf{u})\right] \frac{1}{\mathcal{C} \operatorname{ard}\{U \cap \bar{\Omega}\}} \prod_{i=1}^{d}\left(\mathbf{u}-\mathbf{u}^{\prime}\right)-\frac{\alpha_{d}}{2^{d-1}}
\end{aligned}
$$

where one has $\alpha_{d}=\frac{(d+1) 2^{d-1}}{2^{d}-(d+1)}$ and where the $\widetilde{C}^{(n)}$ notation refers to the functional copula mapping distribution given after renormalization by the truncation according to

$$
\widetilde{C}^{(n)}(B ; \bar{\Omega})=\sum_{\mathbf{u} \in \bar{\Omega} \cap B} \frac{c^{(n)}(\mathbf{u})}{\sum_{\mathbf{u} \in \bar{\Omega}} c^{(n)}(\mathbf{u})}
$$

for all sets $B \in \bar{\Omega}$.

\subsubsection{Understanding Multivariate Spearman's Rank Correlations in Copula Parameter Space}

We illustrate the approximation of the multivariate form of Spearman's rank correlation for a range of different copula models: skewed Student's t-copula; Archimax; and the Clayton copula member of the Archimedean class. Note, we adopt identical parameterizations for these copula models as detailed in Dalessandro and Peters (2016). Furthermore, the accuracy of the reconstruction via the mimicking local Gaussian copula mapping for a general class of copula is detailed in Appendix A and further studied in detail with regard to the 
accuracy of this approximation for each of these specified copula families in Section 3 of Dalessandro and Peters (2016).

The first study we illustrate is to demonstrate the accuracy of our approximation method for evaluation of multivariate Spearman's rank correlation. To achieve this we consider one of the few cases which involves a non-trivial copula model that admits an explicit closed form relationship between the copula parameter and Spearman's rank correlation. This is the 2-dimensional Archimedean copula with the Clayton generator function. We illustrate below in Fig. 3 the exact Spearman's Rho rank correlation as a function of $\theta$ the Clayton copula parameter and we plot our approximation for a range of copula parameter values as crosses. The local Gaussian copula distribution $C^{(n)}$ mimicking the parametric Clayton copula is obtained through the mapping (1.21) on a unit square $\mathbf{U}=\mathbf{U}^{1} \otimes \mathbf{U}^{2}$, with $n_{1}=$ $n_{2}=200$ discretization points. The approximated Spearman's Rho $\rho_{d}^{(n)}\left(C^{(n)} ; \mathbf{U}\right)$ is then computed following proposition 5, where the distribution $C^{(n)}$ is integrated over the set $\mathbf{U}$. The resulting approximated correlation measure is compared with the closed form and it proves to be very accurate.

Next we illustrate how this decomposition can be used to study the behaviour of the copula parameter(s) and the effect varying such parameters will have on the resulting induced concordance measure of interest which in this case will first correspond to the multivariate rank correlation.

In a 2-dimensional illustration, we first consider the skewed Student's t-copula model. We present the case of two different values of skew parameter $\gamma \in\{1,2\}$ and we demonstrate the range of the multivariate Spearman's rho rank correlation as a function of the

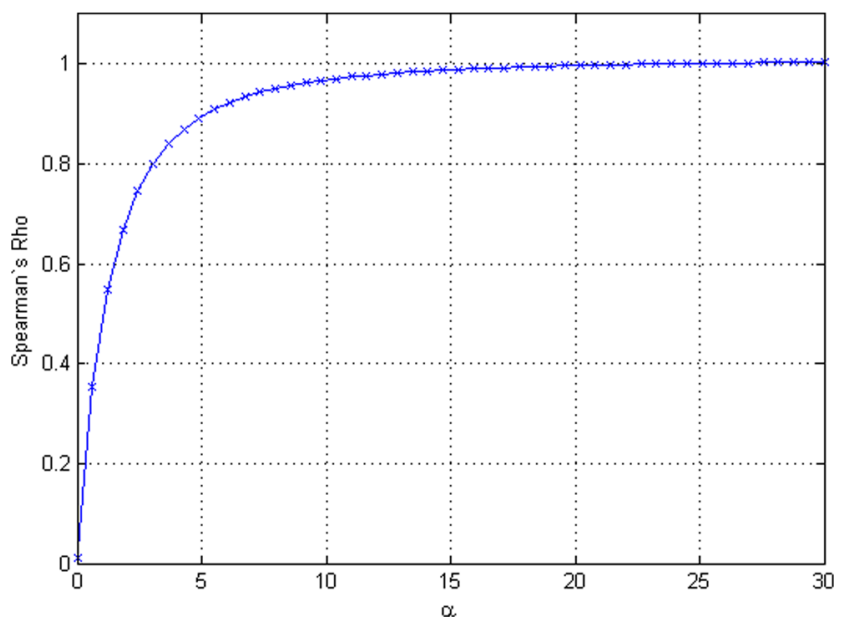

Fig. 3 Spearman's Rho for a Clayton copula with parameter $\alpha$. Solid line is the exact rank correlation as a function of the copula parameter $\alpha$ ranging from $\alpha=0$ to $\alpha=30$, whilst the crosses represent the approximated Spearman's rank correlation $\rho_{d}^{(n)}\left(C^{(n)} ; \mathbf{U}\right)$ obtained using the mapped local Gaussian copula distributions $C^{(n)}$. Several Clayton copula mappings $C_{\text {Clayton }}(\alpha) \mapsto C^{(n)}$ have been calculated each one corresponding to a specific value of the Clayton copula parameter $\alpha \in[0,30]$. The values of the approximated rank correlation $\rho_{d}^{(n)}\left(C^{(n)} ; \mathbf{U}\right)$ coefficients are in line with the closed form ones and they exhibit also the desired asymptotic behaviour $\rho_{d}^{(n)} \rightarrow 1$ as $\alpha \gg 1$ 
degree of freedom parameter $v$ and the correlation parameter $\rho$. Below in Fig. 4 we display the results of the mapping

$$
C_{\text {skewed t-copula }}(\mathbf{u} ;\{\rho, \gamma, v\}) \rightarrow C^{(n)} \rightarrow \rho_{d}^{(n)}\left(C^{(n)} ; \mathbf{U}\right) \text { for } \gamma \in\{1,2\}, \rho \in[-1,1], v \in[10,30]
$$

Firstly, we learn from this analysis that as the skewness parameter $\gamma$ increases, the Spearman's rank correlation surface as a function of correlation $\rho$ and degree of freedom $v$ goes from a concave to a convex relationship. Furthermore, this inversion of the correlation surface in the parameter space of $\rho$ and $v$ is more pronounced at extreme correlations for small degrees of freedom $v$, compared to the Gaussian like tail cases, which begin to arise as $v$ increases. In addition, we see from this analysis that as the skewness parameter $\gamma$ increases, there is a tendency for the model to more readily attain the maximal Spearman's rank correlation of 1 uniformly in the correlation parameter $\rho$ as the degree of freedom parameter $v$ increases. We conjecture from this analysis that as $\gamma \downarrow 0$ the maximal attainable Spearman's rank correlation is strictly less than 1 and ordered in the maximal correlation attained in the parameter $\rho$ for any given $v$.

In the next illustration, see Fig. 5, we demonstrate how the multivariate Spearman rank correlation behaves for the Archimax copula which we constructed using a Pickands function parameterised by $\alpha$ and $\beta$ parameters and a Clayton generator with parameter $\theta$. Even for the 2-dimensional illustration example we present, very little is known about the Spearman's rank correlation for such models as a function of the parameters in this increasingly popular Archimax model. Again, we illustrate the behavior of the Spearman's rank correlation for different fixed values of the Clayton copula parameter, as we vary the Pickands distortion function parameters.

We observe from this study several interesting features. Firstly, there is a lower bound for the Spearman's rank correlation for these models which is uniform across the parameter space for the Pickands distortion function parameters $\alpha$ and $\beta$. Secondly, this lower bound increases in magnitude towards the maximum of 1 as the Clayton generator parameter $\theta$ increases. Thirdly, we observe from this analysis that the maximal values of Spearman's rank correlation for a given combination of $(\alpha, \beta)$ lie on the line $\alpha=\beta$ for any give Clayton generator parameter $\theta$.
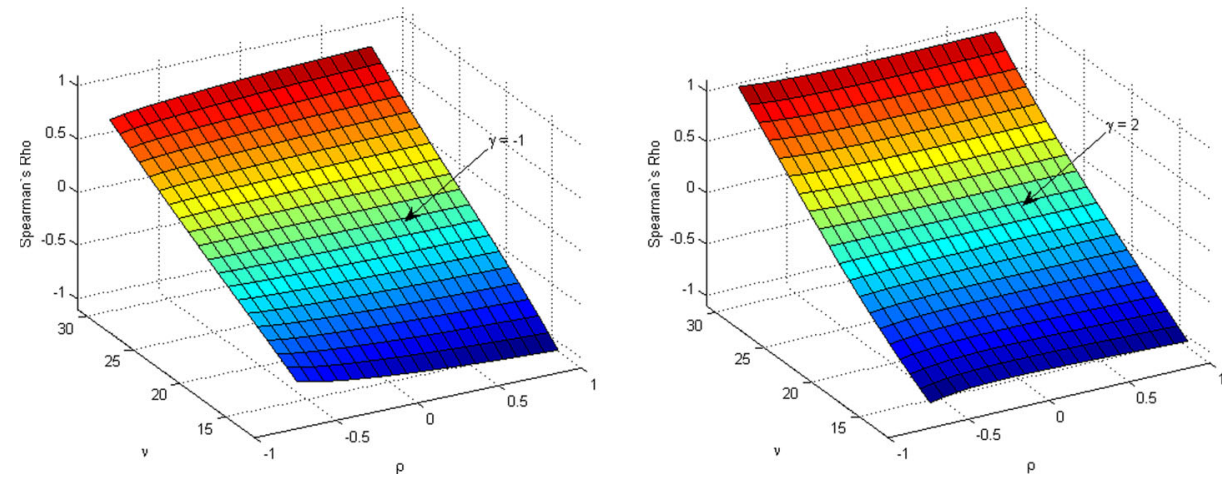

Fig. 4 Approximated Spearman's Rho $\rho_{d}^{(n)}\left(C^{(n)} ; \mathbf{U}\right)$ for a skewed t-copula with parameters $v, \gamma$ and correlation $\rho$ 

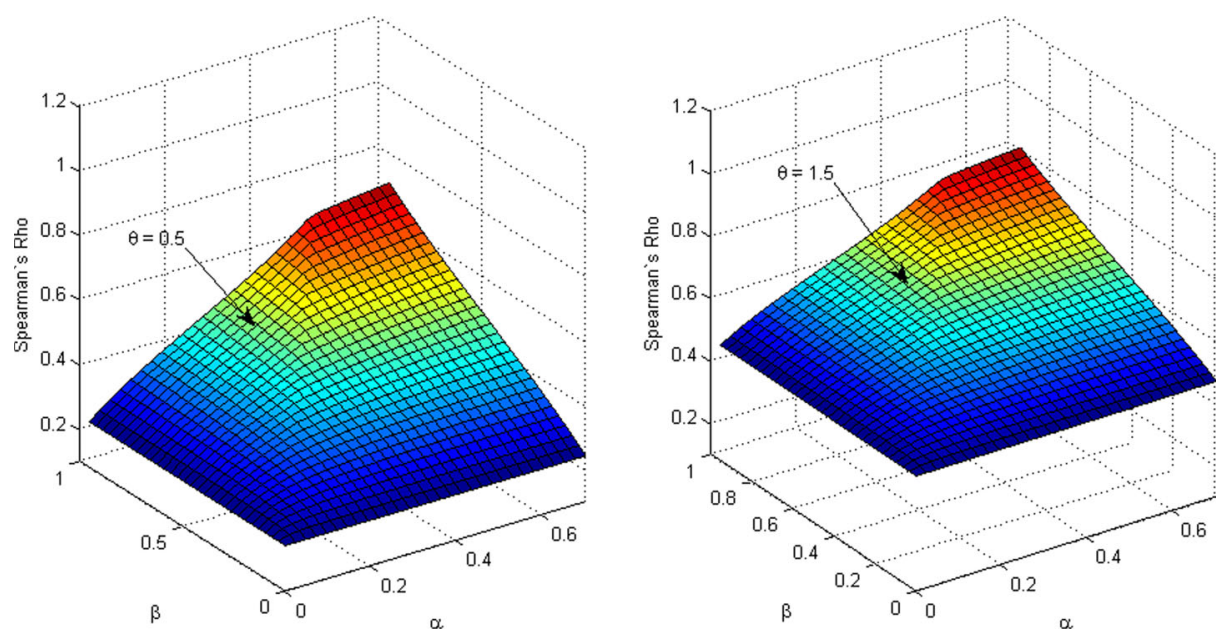

Fig. 5 Approximated Spearman's Rho $\rho_{d}^{(n)}\left(C^{(n)} ; \mathbf{U}\right)$ for a Archimax copula with Pickands parmaters $(\alpha, \beta)$ and Clayton parameter $\theta$

\subsubsection{Multivariate Kendall's Tau Rank Correlation Approximations}

In terms of the multivariate extension of Kendall's tau rank correlation, one can obtain the copula representation of the concept of this rank correlation as follows. Consider the concordance function $\kappa$ quantifying the difference in probabilities of concordance and discordance for bi-variate random vectors $\left(X_{1}, Y_{1}\right)$ and $\left(X_{2}, Y_{2}\right)$ which are specified as follows:

- Assume $X_{1}$ and $X_{2}$ have common continuous marginal $F_{X}$;

- Assume $Y_{1}$ and $Y_{2}$ have common continuous marginal $F_{Y}$;

- Assume $\left(X_{1}, Y_{1}\right)$ and $\left(X_{2}, Y_{2}\right)$ have different copula $C_{1}$ and $C_{2}$ respectively.

Then Nelsen (2002) proposed to consider an alternative copula specified concordance function $\kappa$ for the equivalent Kendall's $\tau$ in a copula form as measuring the probability of concordance and discordance given by

$$
\begin{aligned}
\kappa & =\operatorname{Pr}\left[\left(X_{1}-X_{2}\right)\left(Y_{1}-Y_{2}\right)>0\right]-\operatorname{Pr}\left[\left(X_{1}-X_{2}\right)\left(Y_{1}-Y_{2}\right)<0\right] \\
& =4 \int_{0}^{1} \int_{0}^{1} C_{2}(u, v) d C_{1}(u, v)-1 .
\end{aligned}
$$

Therefore, with this copula based specification, one can then define analogously the functional copula mapping approximation for a given copula for this concordancediscordance copula based Kendall's $\tau$ measure, as well as the local and constrained variants.

Proposition 6 (Approximating the Generalized, Local and Constrained Multivariate Kendall's Tau via Functional Copula Mappings) Consider the $d$-copula given by $C$ which is discretely approximated by the functional copula mapping given by $C^{(n)}(\mathbf{u})$ for $\mathbf{u} \in U$. Hence, one obtains the functional copula approximation for the copula based Kendall's $\tau$ according to

$$
\kappa^{(n)}\left(C^{(n)} ; \mathbf{U}\right)=4\left|\left(u_{1}-u_{1}^{\prime}\right)\left(u_{2}, u_{2}^{\prime}\right)\right| \sum_{\mathbf{u} \in U} C_{2}^{\left(n_{1}, n_{2}\right)}\left(u_{1}, u_{2}\right) c_{1}^{\left(n_{1}, n_{2}\right)}\left(u_{1}, u_{2}\right)-1 .
$$


In addition, the functional approximations to the localized Kendall's $\tau$ for some subspace $\Omega \subseteq \mathbf{U} \subset[0,1]^{d}$ is given according to

$$
\kappa^{(n)}\left(C^{(n)} ; \Omega\right)=4\left|\left(u_{1}-u_{1}^{\prime}\right)\left(u_{2}, u_{2}^{\prime}\right)\right| \sum_{\mathbf{u} \in U \cap \Omega} C_{2}^{\left(n_{1}, n_{2}\right)}\left(u_{1}, u_{2}\right) c_{1}^{\left(n_{1}, n_{2}\right)}\left(u_{1}, u_{2}\right)-1 .
$$

The constrained version of the Kendall's $\tau$ is then given for vectors $u$ constrained in some sub-space $u \in \Omega \subset[0,1]^{d}$ according to

$$
\widetilde{\kappa}^{(n)}\left(C^{(n)} ; \Omega\right)=4\left|\left(u_{1}-u_{1}^{\prime}\right)\left(u_{2}, u_{2}^{\prime}\right)\right| \sum_{\mathbf{u} \in U \cap \Omega} \widetilde{C}_{2}^{\left(n_{1}, n_{2}\right)}\left(u_{1}, u_{2}\right) c_{1}^{\left(n_{1}, n_{2}\right)}\left(u_{1}, u_{2}\right)-1 .
$$

where $\widetilde{C}^{(n)}$ notation refers to the functional copula mapping distribution given after normalization by the truncation, as in Eq. 2.12.

\subsubsection{Understanding Multivariate Kendall's Tau Rank Correlations in Copula Parameter Space}

In this section we illustrate the approximation of the multivariate form of Kendall's tau rank correlation for a range of different copula models: skewed Student's t-copula; Archimax; and the Clayton copula member of the Archimedean class. Again, the first study we illustrate is to demonstrate the accuracy of our approximation method for evaluation of multivariate Kendall's tau rank correlation. To achieve this we consider one of the few cases which involves a non-trivial copula model that admits an explicit closed form relationship between the copula parameter and Kendall's tau rank correlation. This is again the model given by the 2-dimensional Archimedean copula with the Clayton generator function. We illustrate below in Fig. 6 the exact Kendall's Tau rank correlation as a function of $\theta$ the Clayton copula parameter and we plot our approximation for a range of copula parameter values as crosses.

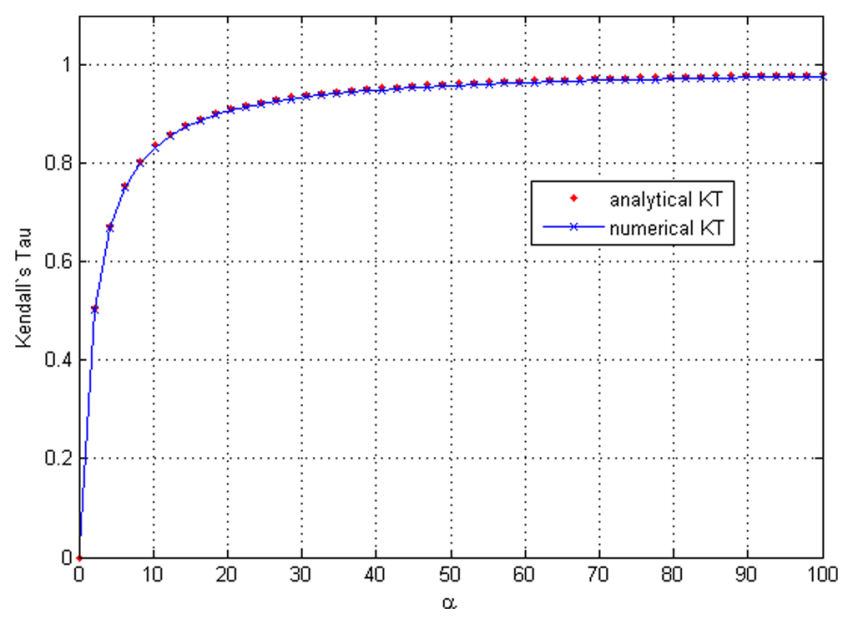

Fig. 6 Kendall's tau for a Clayton copula with parameter $\alpha$. Solid line is the exact rank correlation as a function of copula parameter $\theta$ whilst the crosses represent the approximated Kendall's Tau rank correlation $\kappa^{(n)}\left(C^{(n)} ; \mathbf{U}\right)$ of Eq. 2.15 we obtained via the use of the functional copula mapping summing over the unit square $\mathbf{U}=\mathbf{U}^{1} \otimes \mathbf{U}^{2}$, with $n_{1}=n_{2}=200$ discretization points 
To further illustrate the accuracy of the approximation, see Fig. 7, we construct via the functional copula mapping approach we also include the approximation for the calculation of Kendall's tau coefficient for a second Archimedean copula model given by the generator for the Frank copula, see Nelsen (1999). The copula generator is $\varphi(t)=-\ln \frac{e^{-\theta t}-1}{e^{\theta}-1}$, where $\theta \in \mathbb{R} \backslash\{0\}$. This gives the Frank family

$$
C_{\theta}(u, v)=-\frac{1}{\theta} \ln \left(1+\frac{\left(e^{-\theta u}-1\right)\left(e^{-\theta v}-1\right)}{e^{-\theta}-1}\right)
$$

It can be shown that Kendall's tau is

$$
\tau_{\theta}=1-4 \frac{1-D_{1}(\theta)}{\theta}
$$

where $D_{k}(x)$ is the Debye function given by $D_{k}(x)=\frac{k}{x^{k}} \int_{0}^{x} \frac{t^{k}}{e^{t}-1} d t$, for $k>0$.

\subsubsection{Constructing the Generalized Gaussian Copula Functional Copula for Target Frank Copula}

The Frank copula has generator inverse equal to

$$
-\frac{1}{\theta} \log (1+\exp (-t)(\exp (-\theta)-1))
$$

The density in the $d=3$ case therefore takes the form,

$C\left(u_{1}, u_{2}, u_{3} ; \theta\right)=-\frac{1}{\theta} \log \left(1+\frac{\left(\exp \left(-\theta u_{1}\right)-1\right)\left(\exp \left(-\theta u_{2}\right)-1\right)\left(\exp \left(-\theta u_{3}\right)-1\right)}{(\exp (-t)-1)^{2}}\right), \quad \theta \in \mathbb{R} \backslash\{0\}$.

We demonstrate how one can utilise the copula mapping applied to Eq. 2.21 to study the local effect of dependence induced in different regions of the state space, arising as one

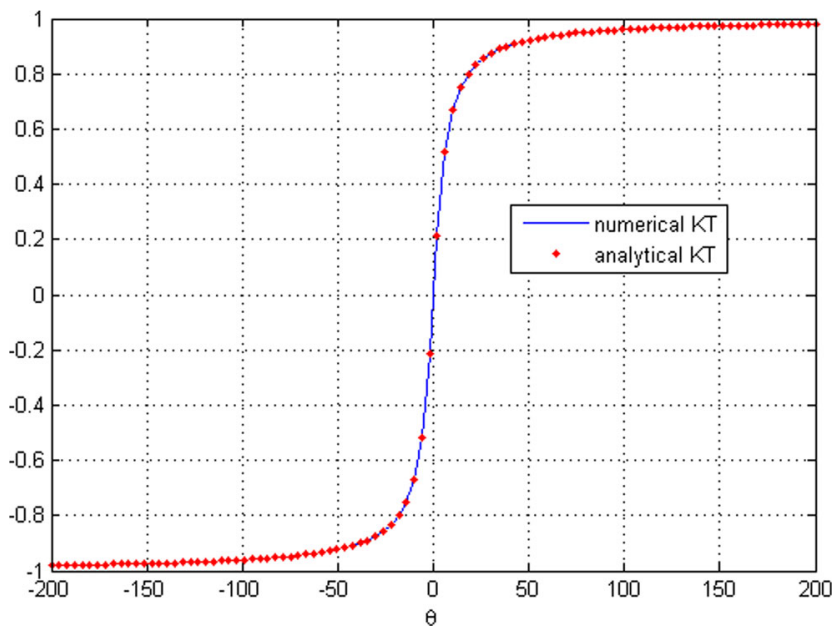

Fig. 7 Approximated Kendall's tau rank correlation $\kappa^{(n)}\left(C^{(n)} ; \mathbf{U}\right)$ of Eq. 2.15 obtained via the use of the functional copula mapping summing over the unit square $\mathbf{U}=\mathbf{U}^{1} \otimes \mathbf{U}^{2}$, with $n_{1}=n_{2}=200$ discretization points for a Frank copula with parameters $\theta \in[-200,200]$ 
varies the parameter $\theta$ of the Frank copula model. Specifically we calculate

$$
\mathbb{E}\left[U_{1}-\mathbb{E}\left[U_{1}\right], U_{1}-\mathbb{E}\left[U_{1}\right], U_{2}-\mathbb{E}\left[U_{2}\right]\right],
$$

and

$$
\mathbb{E}\left[U_{1}-\mathbb{E}\left[U_{1}\right], U_{2}-\mathbb{E}\left[U_{2}\right], U_{3}-\mathbb{E}\left[U_{3}\right]\right],
$$

which can be computed using (Figs. 8, 9 and 10)

$$
\sum_{\mathbf{u}} c^{(n)}(\mathbf{u})(\mathbf{u}-\hat{\mathbf{u}}), \text { for all } \mathbf{u} \in \mathbf{U}=\bigotimes_{k=1}^{3} \mathbf{U}^{k},
$$

or equivalently

$\sum_{u^{(1)}} \sum_{u^{(2)}} \sum_{u^{(3)}} c^{(n)}\left(u^{(1)}, u^{(2)}, u^{(3)}\right)\left(u^{(1)}-\hat{u}, u^{(2)}-\hat{u}, u^{(3)}-\hat{u}\right)$, for all $\mathbf{u}=\left(u^{(1)}, u^{(2)}, u^{(3)}\right) \in \mathbf{U}, \hat{u}=\frac{1}{2}$.

Next, as with the case of the Spearman's Rho rank correlation, we now repeat the studies of the Kendall's tau approximation to the multivariate rank correlation for the skewed Student's t-copula and the Archimax copula comprised of Clayton generator and Pickands' distortion function, the results are presented in Figs. 11 and 12.

\subsection{Multivariate Directional Dependence Approximations}

After considering these multivariate copula specifications of rank correlations, one can also consider copula specifications of notions of directional dependence as detailed in for instance the notions of intermediate directional dependence in the 3-dimensional context, see details in Nelsen (2002). We will denote such dependence measures as rho-directional dependence, say in for instance the 3-dimensional setting are then easily obtained using these functional copula approximations of the rank correlations as follows.

Proposition 7 (Functional Copula Approximations for 3-Copula $\rho$-Directional Dependence) Let $C(\mathbf{u}), \mathbf{u}=\left(u_{1}, u_{2}, u_{3}\right)$ a 3-dimensional copula distribution function with
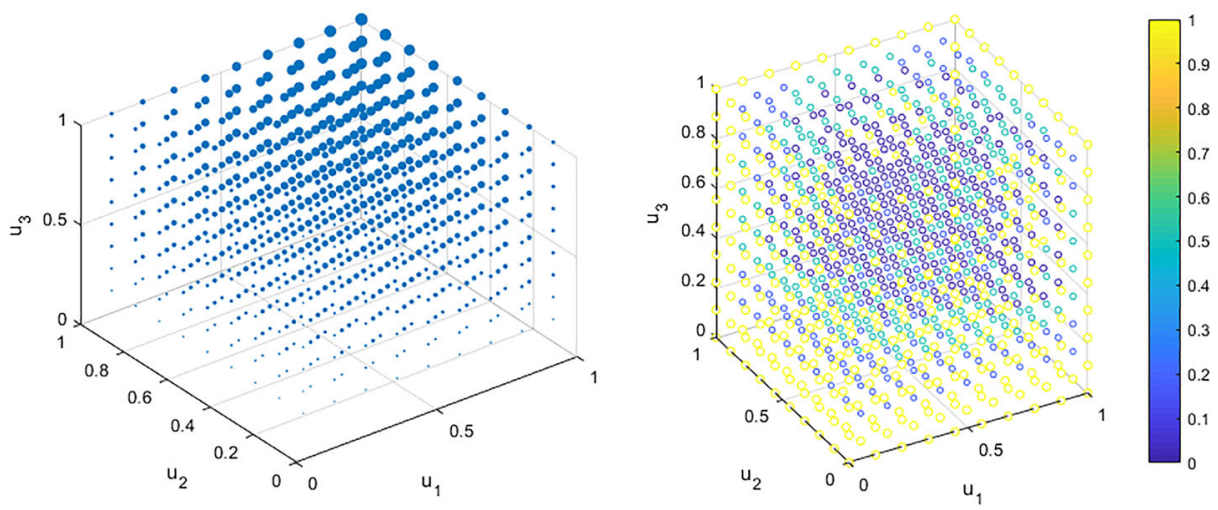

Fig. 8 The left plot reports the approximated 3-dimensions Frank copula distribution $C: \mathbf{U}=\bigotimes_{k=1}^{3} \mathbf{U}^{k} \rightarrow$ $[0,1]$ as per (2.21), with parameter $\theta=6$ and $n_{1}=n_{2}=n_{3}=20$. The scatter plot on the right report the mapped local Gaussian correlation $\rho(\mathbf{u}) \in[-1,1]$, for all $\mathbf{u} \in \mathbf{U}$. Note that for a Frank copula parameter $\theta=6$, we have only positive intermediate tail dependence, which is evident after performing the local Gaussian mapping 

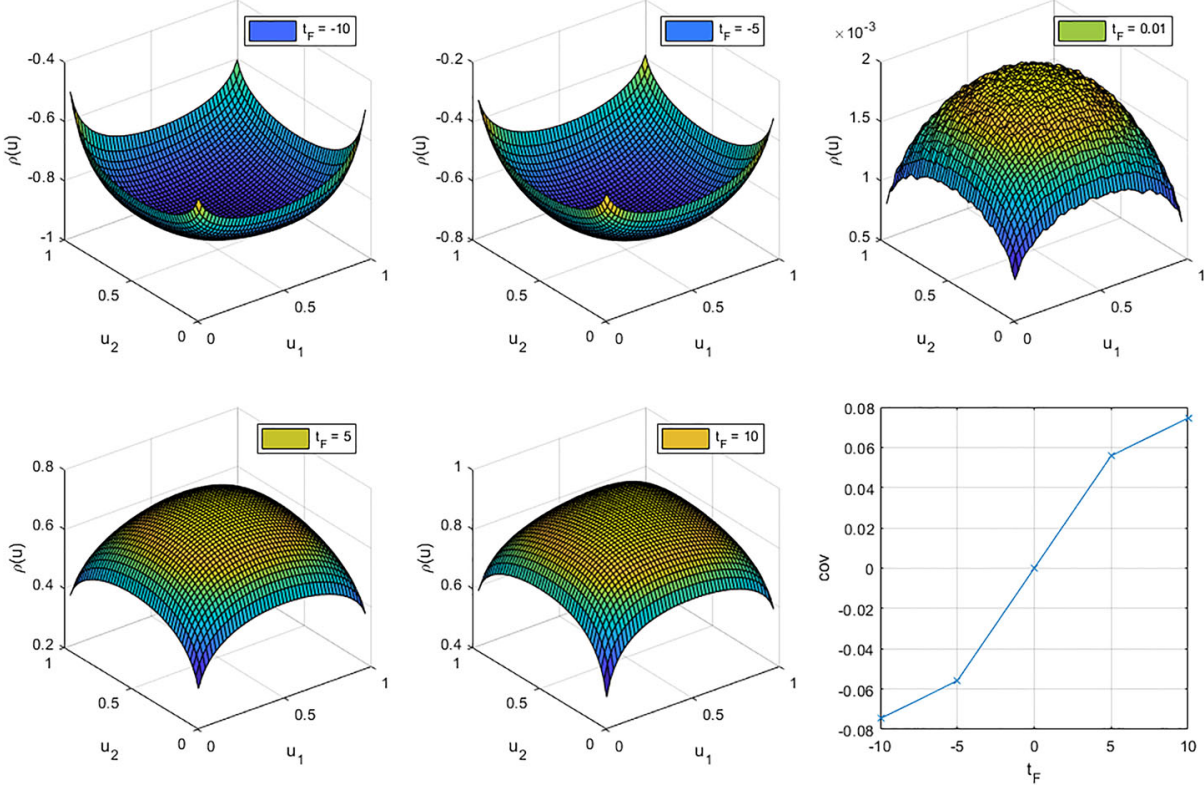

Fig. 9 The plots report the mapped local Gaussian correlation $\rho(\mathbf{u}): \bigotimes_{k=1}^{2} \mathbf{U}^{k} \rightarrow[-1,1]$ for two dimensional Frank copulas with parameters $\theta=\{-10,-5,0.01,5,10\}$. It is evident the symmetry of the local Gaussian correlation when the Frank copula parameter $\theta$ has opposite sign and this is confirmed in the last bottom right plot where we report the Frank copula covariance cov $=\mathbb{E}\left[\left(U_{1}-\mathbb{E}\left[U_{1}\right]\right)\left(U_{2}-\mathbb{E}\left[U_{2}\right]\right)\right]$. The number of discretization points used in this calculation is $n_{1}=n_{2}=200$

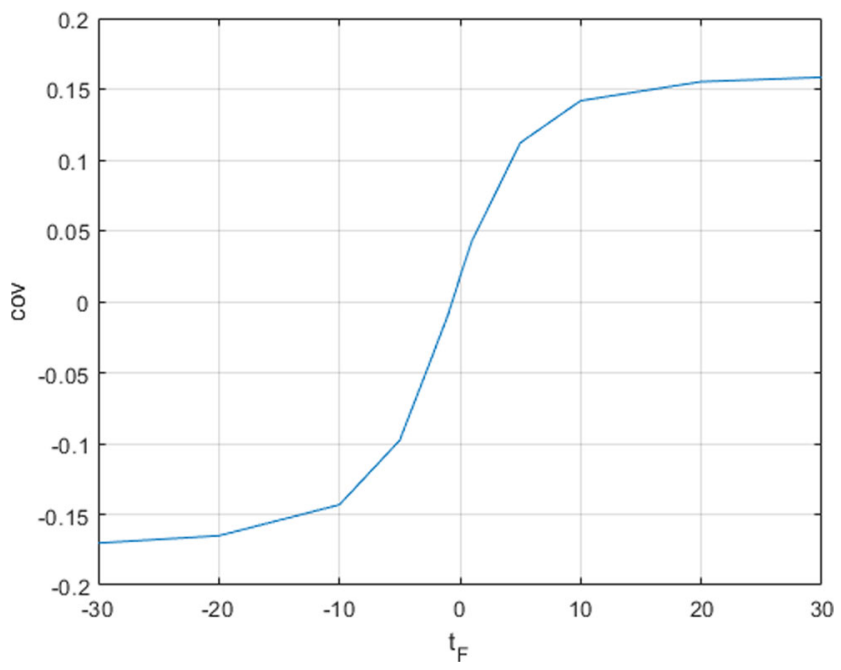

Fig. 10 The plot reports the behaviour of the covariance for a mapped 3-dimensions Frank copula with parameters $\theta=\{-10,-5,0.01,5,10\}$. The covariance cov $=\mathbb{E}\left[\left(U_{1}-\mathbb{E}\left[U_{1}\right]\right)\left(U_{2}-\mathbb{E}\left[U_{2}\right]\right)\left(U_{3}-\mathbb{E}\left[U_{3}\right]\right)\right]$ is computed using Eq. 2.25. The covariance reflects the symmetry of the Frank copula. The number of discretization points used in this calculation is $n_{1}=n_{2}=n_{3}=200$ 

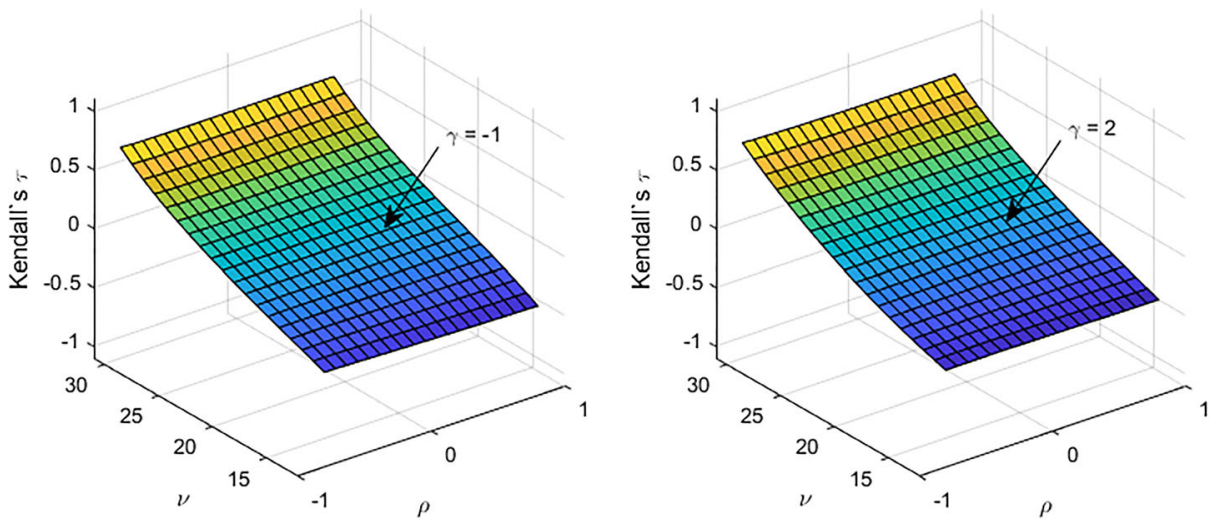

Fig. 11 Approximated Kendall's tau $\kappa^{(n)}\left(C^{(n)} ; \mathbf{U}\right)$ for a skewed t-copula with parameters $v, \gamma$ and correlation $\rho$. The computation of Eq. 2.15 has been done over the unit square $\mathbf{U}=\mathbf{U}^{1} \otimes \mathbf{U}^{2}$, with $n_{1}=n_{2}=200$ discretization points

continuous marginals $X=\left(X_{1}, X_{2}, X_{3}\right)$ uniform on $[0,1]^{3}$. Then for any direction $\left(\alpha_{1}, \alpha_{2}, \alpha_{3}\right)$ characterised by the vector components $\alpha_{i} \in\{-1,1\}$ for $i \in\{1,2,3\}$, one has the $\rho$-directional dependence, expressed by a discretized copula function $C^{(n)}$, is given by

$$
\begin{aligned}
& \rho_{X_{1}, X_{2}, X_{3}}^{\left(n_{1}, n_{2}, n_{3}\right)\left(\alpha_{1}, \alpha_{2}, \alpha_{3}\right)}=\frac{\alpha_{1} \alpha_{2} \rho_{X_{1}, X_{2}}^{\left(n_{1}, n_{2}\right)}+\alpha_{2} \alpha_{3} \rho_{X_{2}, X_{3}}^{\left(n_{2}, n_{3}\right)}+\alpha_{3} \alpha_{1} \rho_{X_{3}, X_{1}}^{\left(n_{3}, n_{1}\right)}}{3} \\
& +\alpha_{1} \alpha_{2} \alpha_{3} \frac{\left[\rho_{X_{1}, X_{2}, X_{3}}^{\left(n_{1}, n_{2}, n_{3}\right)}\right]^{+}-\left[\rho_{X_{1}, X_{2}, X_{3}}^{\left(n_{1}, n_{2}, n_{3}\right)}\right]^{-}}{2}
\end{aligned}
$$
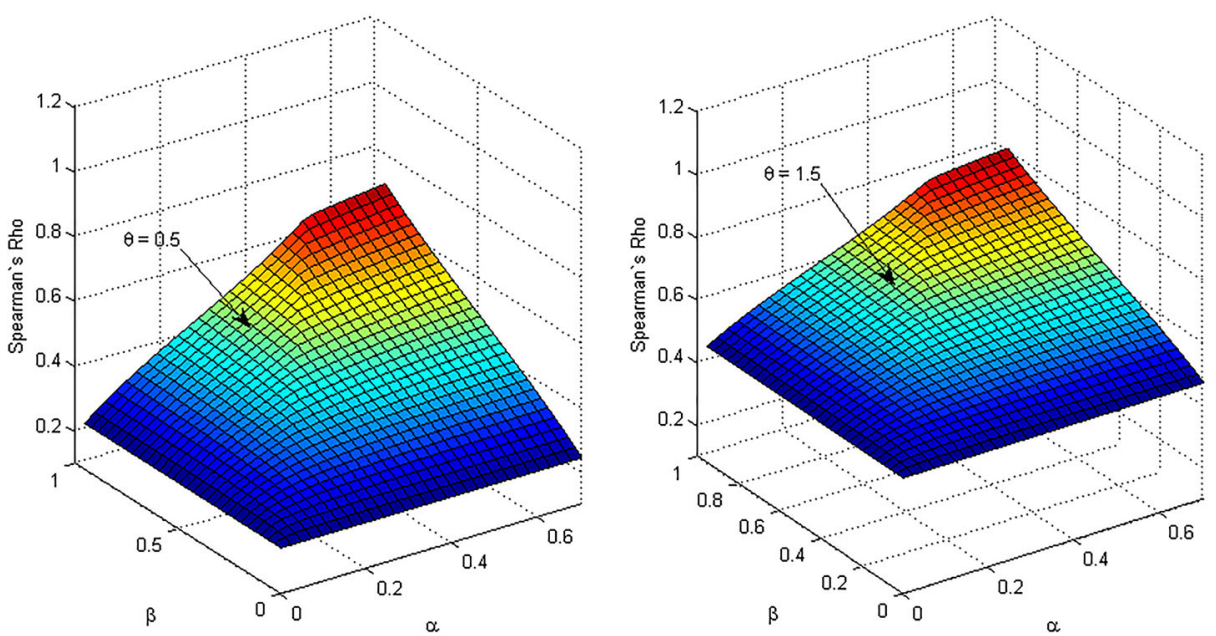

Fig. 12 Approximated Kendall's tau $\kappa^{(n)}\left(C^{(n)} ; \mathbf{U}\right)$ for an Archimax copula with Pickands parameters $(\alpha, \beta)$ and Clayton parameter $\theta$. The computation of Eq. 2.15 has been done over the unit square $\mathbf{U}=\mathbf{U}^{1} \otimes \mathbf{U}^{2}$, with $n_{1}=n_{2}=200$ discretization points 

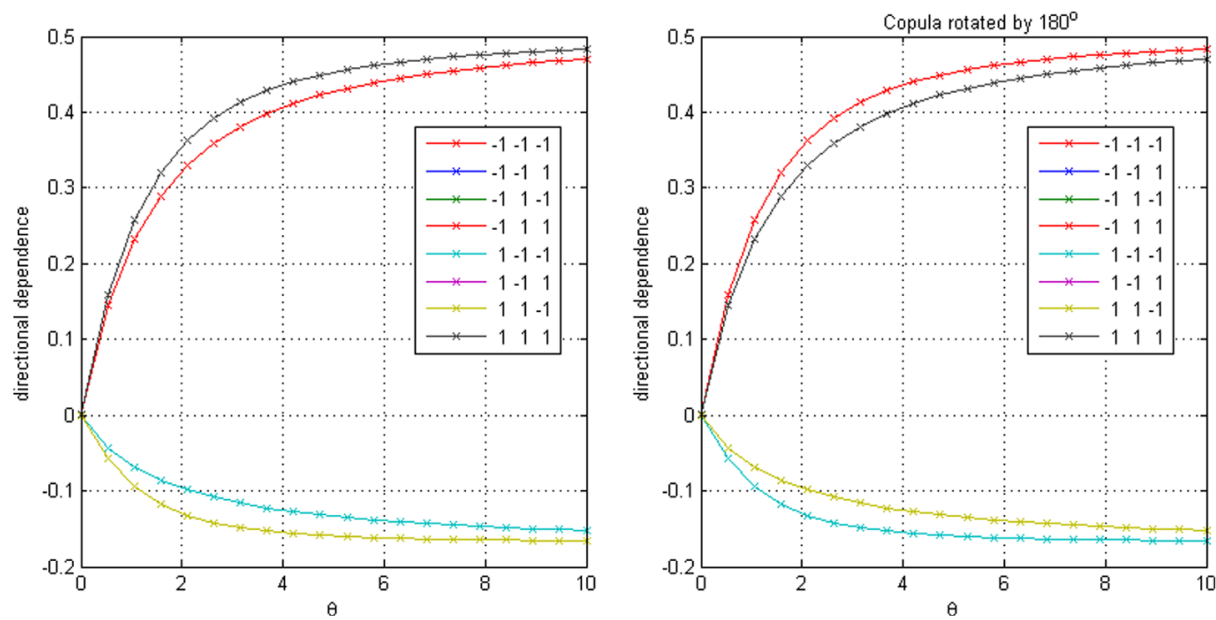

Fig. 13 Approximated Spearman's Rho directional dependence for a 3-D Clayton copula with parameter $\theta$ computed using Eq. 2.27, over the unit cube $\mathbf{U}=\bigotimes_{i=1}^{3} \mathbf{U}^{i}$, with $n_{1}=n_{2}=n_{3}=60$. The plot on the left displays the set of directional dependence curves for a standard 3-D Clayton copula, each of them is a different permutation of three distinct direction coefficients $\left(\alpha_{1}, \alpha_{2}, \alpha_{3}\right)$. The plot on the right reports directional dependence curves for a 3-D Clayton copula rotated by $180^{\circ}$. The rotation of the copula imposes a change in tail dependence that is accurately reflected by the directional dependence curves. This behaviour is shown in the above plots and specifically by the red and black curves, which interchange values

with pairwise Spearman's rho obtained via the functional copula approximations given above in Proposition 5 and

$$
\begin{aligned}
& {\left[\rho_{X_{1}, X_{2}, X_{3}}^{\left(n_{1}, n_{2}, n_{3}\right)}\right]^{+}\left(C^{\left(n_{1}, n_{2}, n_{3}\right)}\right)=8\left|\prod_{i=1}^{3}\left(u_{i}-u_{i}^{\prime}\right)\right| \sum_{u \in U \cap \Omega} \bar{C}^{\left(n_{1}, n_{2}, n_{3}\right)}(u)-1,} \\
& {\left[\rho_{X_{1}, X_{2}, X_{3}}^{\left(n_{1}, n_{2}, n_{3}\right)}\right]^{-}\left(C^{\left(n_{1}, n_{2}, n_{3}\right)}\right)=8\left|\prod_{i=1}^{3}\left(u_{i}-u_{i}^{\prime}\right)\right| \sum_{u \in U \cap \Omega} C^{\left(n_{1}, n_{2}, n_{3}\right)}(u)-1 .}
\end{aligned}
$$

Remark 2.1 The eight vectors which characterize directions $\left(\alpha_{1}, \alpha_{2}, \alpha_{3}\right)$ where $\alpha_{i} \in$ $\{-1,1\}$ for $i \in\{1,2,3\}$ in $[0,1]^{3}$ allow one to utilise the $\rho$-directional dependence to measure directional dependence in different quadrants (Fig. 13).

\subsection{General Copula Approximations for Intermediate and Asymptotic Tail Dependence}

The joint behaviour of extremes for a multivariate copula distribution often are directly influenced through a non-trivial and often non-linear relationship between the copula parameter(s), the dimension and the choice of copula and direction in which extremes are considered. For instance, if we consider extremes in the direction of some angle $\theta$, special cases often get singled out, such as $\theta \in\{\pi / 4,5 \pi / 4\}$ which correspond to particular types of quadrant dependence and are typically known as upper and lower intermediate and asymptotic tail dependencies.

Tail dependence provides one approach to quantification of the dependence in extremes of a multivariate distribution. Traditionally this notion of dependence was considered from a pairwise construction due to tractability of expressions for the pairwise construction when 
applied to copula models. However there is no reason to restrict this notion to just pairwise analysis and below we consider first the pairwise definition and then the generalized definition for $d$-variate random vectors.

In addition, there is also interest in studying related functions known as tail dependence functions and tail orders. In some cases the tail dependence has been specified, for instance by De Luca and Rivieccio (2012) and Li (2009), according to the formulation where for an arbitrary d-variate cases, such that $d>2$ one may quantify the tail dependence present between sub-vector partitions of the multivariate random vector with regard to joint tail dependence behaviours. Such a specification can be made as shown in Definition 12.

Definition 12 (Multivariate Tail Dependence) Let $\mathbf{X}=\left(X_{1}, \ldots, X_{d}\right)$ be a d-dimensional random vector with marginal distribution functions $F_{1}, \ldots, F_{d}$ and copula $\mathrm{C}$.

1. One may define the coefficient of multivariate upper tail dependence (upper orthant dependence) by:

$$
\begin{aligned}
\lambda_{u}^{1, \ldots, h \mid h+1, \ldots, d} & =\lim _{\nu \rightarrow 1-} P\left(X_{1}>F^{-1}(v), \ldots, X_{h}>F^{-1}(v) \mid X_{h+1}>F^{-1}(v), \ldots, X_{d}>F^{-1}(v)\right) \\
& =\lim _{\nu \rightarrow 1-} \frac{\bar{C}_{n}(1-v, \ldots, 1-v)}{\bar{C}_{n-h}(1-v, \ldots, 1-v)}
\end{aligned}
$$

where $\bar{C}$ is the survival copula of $\mathrm{C}$.

2. One may define the coefficient of multivariate lower tail dependence (lower orthant dependence) by:

$$
\begin{aligned}
\lambda_{l}^{1, \ldots, h \mid h+1, \ldots, d} & =\lim _{\nu \rightarrow 0+} P\left(X_{1}<F^{-1}(v), \ldots, X_{h}<F^{-1}(v) \mid X_{h+1}<F^{-1}(v), \ldots, X_{d}<F^{-1}(v)\right) \\
& =\lim _{\nu \rightarrow 0+} \frac{C_{n}(v, \ldots, v)}{C_{n-h}(v, \ldots, v)}
\end{aligned}
$$

where, $\mathrm{h}$ is the number of variables conditioned on (from the $\mathrm{d}$ considered).

Alternative definitions of multivariate tail dependence are also popular in the literature, for instance the specification given in Klüppelberg et al. (2008) where they define the tail dependence function according to Definition 13.

Definition 13 (Tail Dependence Function of a Multivariate Distribution) Consider a $d$ dimensional random vector $\mathbf{X}=\left(X_{1}, \ldots, X_{d}\right) \in \mathbb{R}^{d}, d \geq 2$ with marginal distribution functions $F_{1}, \ldots, F_{d}$, then the tail dependence function is given by

$$
\lambda(\mathbf{u}):=\lambda\left(x_{1}, x_{2}, \ldots, x_{d}\right)=\lim _{t \rightarrow 0} \frac{1}{t} \operatorname{Pr}\left[\bar{F}_{1}\left(X_{1}\right) \leq t x_{1}, \ldots, \bar{F}_{2}\left(X_{d}\right) \leq t x_{d}\right], \quad \mathbf{x} \in \mathbb{R}^{d} .
$$

In Joe et al. (2010) they studied properties of tail dependence functions and conditional tail dependence functions, which they defined via the joint distribution on the unit d-dimensional hyper-cube known as a copula, for a multi-variate distribution. The definition adopted in Joe et al. (2010) for the upper and lower tail dependence functions differs to that provided in Definition 13 via the fact that each marginal can go to the limit at different rates according to the functions: 
- Lower Tail Dependence Function: the tail dependence function for the copula distribution $C\left(u_{1}, \ldots, u_{d}\right)$ is given by

$$
\lambda_{l}(t ; C(\mathbf{u}))=\lim _{t \downarrow 0} \frac{C\left(t u_{1}, \ldots, t u_{d}\right)}{t}, \forall u=\left(u_{1}, \ldots, u_{d}\right) \in \mathbb{R}_{+}^{d}
$$

- Upper Tail Dependence Function: the tail dependence function for the copula distribution $C\left(u_{1}, \ldots, u_{d}\right)$ is given by

$$
\lambda_{u}(t ; C(\mathbf{u}))=\lim _{t \uparrow 1} \frac{\bar{C}\left(t u_{1}, \ldots, t u_{d}\right)}{t}, \forall u=\left(u_{1}, \ldots, u_{d}\right) \in \mathbb{R}_{+}^{d}
$$

where we denote by $\bar{C}\left(u_{1}, \ldots, u_{d}\right)=C\left(1-u_{1}, \ldots, 1-u_{d}\right)$ the survival copula distribution.

The existence of such limits in the definition of the tail dependence functions can be linked to existence of multivariate regular variation on the copula distribution tails.

Given these definitions, one may now define the following intermediate and extremal tail dependence measures

Definition 14 (Intermediate and asymptotic Directional Tail Dependence Functions) Consider the $d$-dimensional random vector $\mathbf{X}=\left(X_{1}, X_{2}, \ldots, X_{d}\right) \in \mathbb{R}^{d}$ with joint distribution characterized by marginals $F_{i}\left(x_{i}\right)$ and joint copula distributions $C\left(F_{1}\left(x_{1}\right), \ldots, F_{d}\left(x_{d}\right)\right)$. The intermediate tail dependence function for a $k$-dimensional sub-vector $y, k \leq d$ for some angular region $\Omega=\prod_{i=1}^{d-1}\left[\theta_{i}, \theta_{i}^{\prime}\right] \subseteq \prod_{i=1}^{d-2}[0, \pi] \times[0,2 \pi]$ and a radius $r$ by

$$
\tilde{\lambda}(x, k, d, \Omega)=\operatorname{Pr}\left[X_{1} \leq F_{1}^{-1}(u(r)), \ldots, X_{k} \leq F_{k}^{-1}(u(r)) \mid \mathcal{H}(x, k, d, \Omega)\right]
$$

where $\mathcal{H}(x, k, d, \Omega) \quad:=\quad\left\{X_{k+1} \leq F_{k+1}^{-1}(u(r)), \ldots, X_{d} \leq F_{d}^{-1}(u(r))\right\}$

$\left\{x \in \mathcal{C}_{d-1}(\theta \in \Omega)\right\}$. The asymptotic tail dependence coefficient is then given by the limiting behaviour of this intermediate tail dependence function according to

$$
\lambda(k, d, \Omega)=\lim _{r \rightarrow \infty} \operatorname{Pr}\left[X_{1} \leq F_{1}^{-1}(u(r)), \ldots, X_{k} \leq F_{k}^{-1}(u(r)) \mid \mathcal{H}(x, k, d, \Omega)\right]
$$

As with the other concordance measures, we may also approximate these measures using the developed functional copula mapping approach. In the following example we illustrate the behaviour of the tail dependence function specified directly above, in the 2-dimensional case of the Clayton copula.

If we consider the two dimensional case with random variables $X_{1}$ and $X_{2}$ with distributions $F_{i}, i=1,2$ and a density describing their dependence on the unit cube known as a copula $C$. Then we may define for $\Omega=[0, \pi / 2]$ the upper tail dependence coefficient by:

$$
\lambda_{u}:=\lim _{u \uparrow 1} \operatorname{Pr}\left[X_{2}>F_{2}^{-1}(u) \mid X_{1}>F_{1}^{-1}(u)\right]=\lim _{u \uparrow 1} \frac{1-2 u+C(u, u)}{1-u}
$$

and similarly we define for $\Omega=[-\pi,-\pi / 2]$ the coefficient of lower tail dependence given by:

$$
\lambda_{l}:=\lim _{u \downarrow 0} \operatorname{Pr}\left[X_{2} \leq F_{2}^{-1}(u) \mid X_{1} \leq F_{1}^{-1}(u)\right]=\lim _{u \downarrow 0} \frac{C(u, u)}{u}
$$

Note that $\widetilde{C}(1-u, 1-u)=1-2 u-C(u, u)$ is known the survival copula of $C$. The above relationships show that the upper tail dependence coefficients of copula $C$ is also equal to the lower tail dependence coefficient of the survival copula of $C$. Analogously, the lower tail 


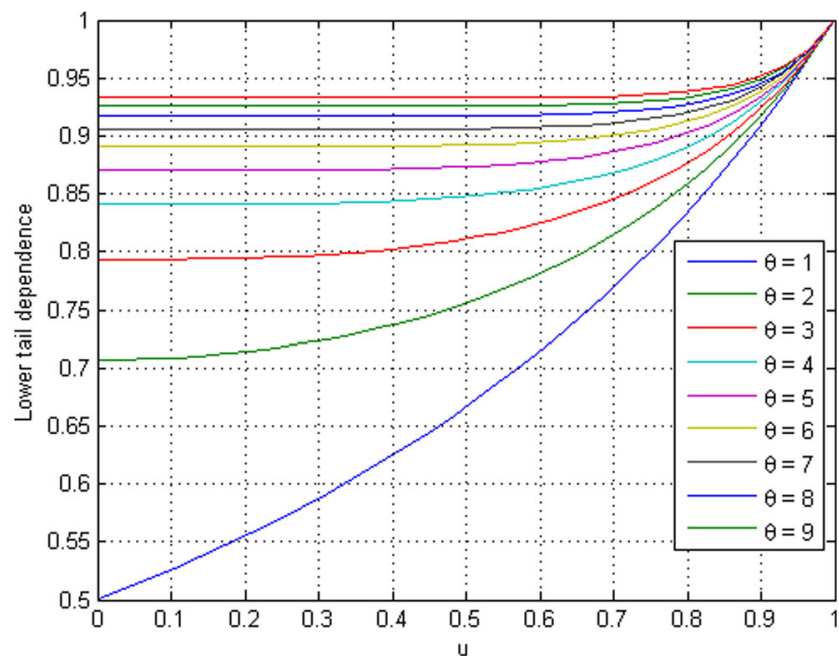

Fig. 14 Intermediate and asymptotic approximated lower tail (LT) coefficient $\lambda_{l}^{(n)}\left(C^{(n)}\right)$ for a Clayton copula with parameter $\theta$ computed using Eq. 2.37. For $u \rightarrow 0$ the value of the LT coefficient correspond to the asymptotic one

dependence coefficient of copula $C$ is equivalent to the upper tail dependence coefficient of the survival copula.

Remark 2.2 Similar to rank correlations, the tail dependence coefficient is a simple scalar measure of dependence that depends on the copula of two random variables but not on their marginal distributions.

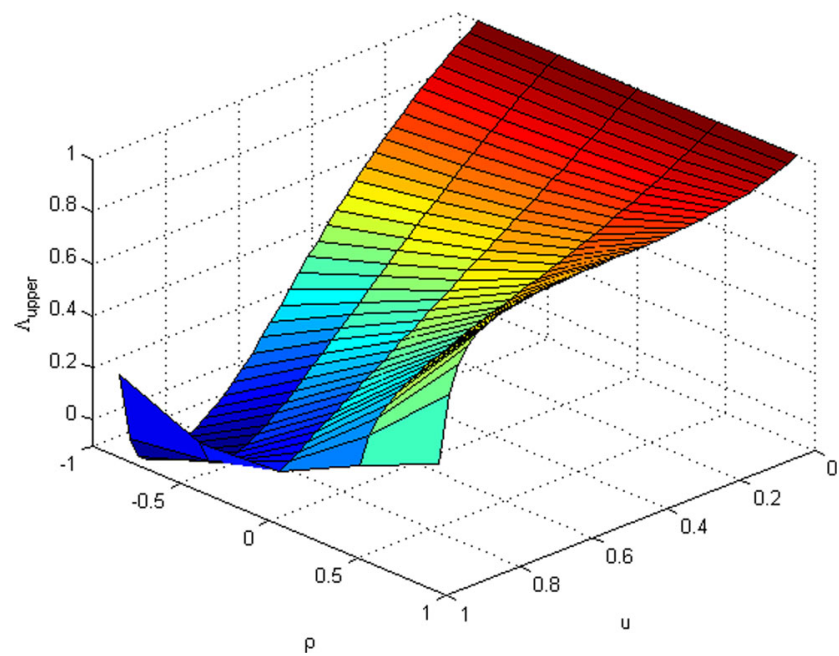

Fig. 15 Intermediate and asymptotic approximated upper tail (UT) coefficient $\lambda_{u}^{(n)}\left(C^{(n)}\right)$ for a skewed Student's t-copula with parameters $g=-1, v=11$ and correlation parameter $\rho \in[-1,1]$, computed using Eq. 2.38. For $u \rightarrow 1$ the value of the UT coefficient corresponds to the asymptotic one 


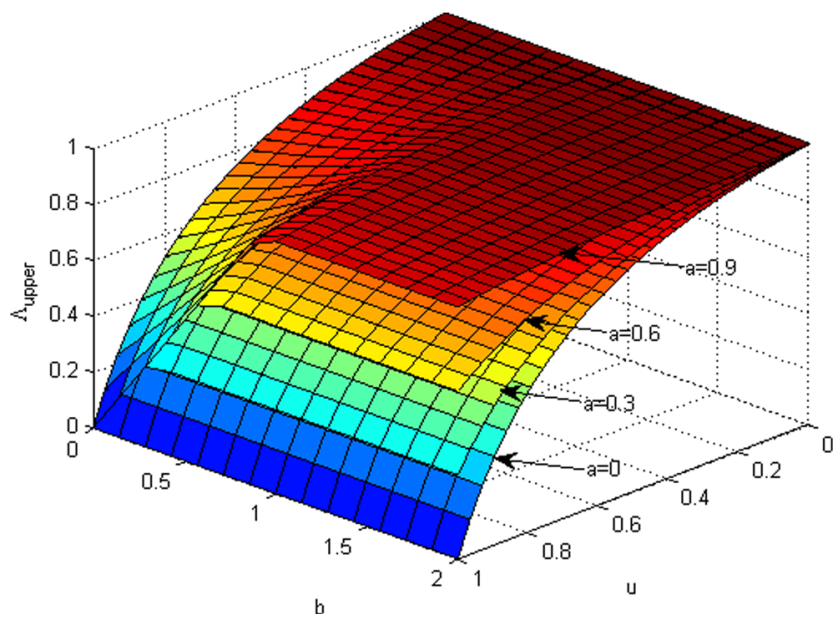

Fig. 16 Intermediate and asymptotic upper tail (UT) coefficient for a 2-D Archimax Copula with Clayton generating function $\varphi(x, \theta)$ and Pickands function $\mathcal{A}(t)$. The parameters are $\theta=3, a=[0,0.3,0.6,0.9]$ and $b \in[0,2]$. Note that when $(a, b)=(0,0)$ then the Archimax copula is equivalent to the Clayton one with the same $\theta$ parameter that is equal to 3 in this specific example. For $u \rightarrow 1$ the value of the UT coefficient corresponds to the asymptotic one

Following we show how it is possible to compute coefficients of intermediate tail dependence using the copula approximation framework proposed in the previous section. After selecting few representative target copula functions we use the mapping of Eq. 1.10 to obtain the approximated copula distributions $C^{(n)}$ which are function of the local Gaussian correlations. In the examples we used $n=200$ as a number of discretization point across the dimensions of the unit hypercube, which is the support of the mapped and discretized copula distribution functions. Specifically in Fig. 14 we calculate the approximated intermediate and asymptotic lower tail directional dependence

$$
\lambda_{l}^{(n)}\left(C^{(n)}\right)=\frac{C^{(n)}(\mathbf{u}, \mathbf{u})}{\mathbf{u}}, \mathbf{u}=\mathbf{0}, \ldots, \mathbf{1},
$$

for a mapped Clayton copula $C^{(n)}$ with parameters $\theta=\{1,2,3,4,5,6,7,8$, 9\}. In Fig. 15 we calculate the intermediate and asymptotic upper tail for a skewed Student's t-copula with parameters $\rho \in[-1,1], v=11$ and $g=-1$. In Fig. 16 we calculate the approximated intermediate and asymptotic upper tail (UT) coefficient

$$
\lambda_{u}^{(n)}\left(C^{(n)}\right)=\frac{1-2 \mathbf{u}-C^{(n)}(\mathbf{u}, \mathbf{u})}{1-\mathbf{u}}, \mathbf{u}=\mathbf{0}, \ldots, \mathbf{1},
$$

for an approximated two dimensional mapped Archimax Copula $C^{(n)}$ with Clayton generating function $\varphi(x, \theta)$ and Pickands function $\mathcal{A}(t)$ with parameters $\theta=3, a=$ $\{0,0.3,0.6,0.9\}$ and $b \in[0,2]$. The examples highlight how the proposed framework can deal with complex dependence structure like the case of the Clayton copula with Archimax distortion function. 


\section{Conclusions}

In this paper we presented a numerical procedure to map any copula function to a generalized Gaussian copula function. This allows us to visualize a given copulas' characteristics and better quantify the local correlation generated by the copula function. In fact copulas' parametric expressions are neither intuitive nor do they reflect the dependence structure that they create. The proposed copula mapping is entirely based on tensor algebra and is part of a new modelling framework we also introduced consisting of the tensor approximation of the infinitesimal generator associated to multidimensional correlated diffusions. We demonstrated the simplicity and intuition behind the copula mapping through illustrative examples and all the copula functions mapping results are exact up to the tensor space local discretization error. We proposed a class of new methods that involves a highly accurate and computationally efficient procedure to evaluate concordance measures for a given copula, applicable even when sampling from the copula is not easily achieved. In addition, this then allows us to reconstruct maps of concordance measures locally in all regions of the state space for any range of copula parameters. We believe this technique will be a valuable tool for practitioners to understand better the behaviour of copula models and associated concordance measures expressed in terms of these copula models and also to capture, measure and quantify different notions of dependence in stochastic processes.

Open Access This article is distributed under the terms of the Creative Commons Attribution 4.0 International License (http://creativecommons.org/licenses/by/4.0/), which permits unrestricted use, distribution, and reproduction in any medium, provided you give appropriate credit to the original author(s) and the source, provide a link to the Creative Commons license, and indicate if changes were made.

\section{Appendix A: Background Results on Reconstruction of a Copula via a Tensor Decomposition}

This appendix provides a summary of the key results and algorithms from Dalessandro and Peters (2017), which include:

1. Construction of a process mimicking a diffusion through local moment matching. At this purpose the mimicking process is a birth-death process (BDP), and we show how to calculate its birth and death rate in a way to exactly replicate some target moments. The BDP is represented through a continuous time Markov chain (CTMC).

2. Construction of a process mimicking a conditional diffusion. At this purpose we introduce the concept of conditional infinitesimal generator matrix, which is a sequence of generator matrices. We also introduce the concept of orthogonal projections in a tensor space.

3. Approximation of the weak solution of a diffusion through the matrix exponentiation of the infinitesimal generator matrix.

4. Approximation of the weak solution of a multidimensional diffusion through the matrix exponentiation of an infinitesimal generator matrix which spans a tensor Kronecker product space. The weak solution is approximated only over orthogonal dimensions and this means that the multivariate weak solution is not directly constructed rather calculated from elementary one dimensional conditional weak solutions.

Let $\mathbf{X}_{t}, t \geq 0$ a $d$ dimensional Markov process with $\left(X_{t}^{1}, \ldots, X_{t}^{d}\right) \in \mathbb{R}^{d}$ denoting its components vector at time $t$, with $\mathbb{R}^{d}$ denoting the d-dimensional Euclidean space. Our 
modelling framework deals with the local approximation of $X_{t}$ by birth-death processes (BDP) and their associated continuous time Markov chains (CTMC).

It suffices, in the methods required for this manuscript, to restrict our attention to the analysis of continuous Markov processes with stochastic differential equation (SDE) of the form,

$$
d \mathbf{X}_{t}=\mathbf{b}\left(\mathbf{X}_{t}\right) d t+\Psi\left(\mathbf{X}_{t}\right) d \mathbf{W}_{t}
$$

where $\mathbf{b}(\mathbf{x})=\left(b_{i}(\mathbf{x})\right), i=1, \ldots, d$ is a drift vector function with $\mathbf{b}(\mathbf{x}): \mathbb{R}^{d} \rightarrow \mathbb{R}^{d}$ and $\Sigma=\Psi \Psi^{\prime}$ is a covariance matrix continuous function, with $\Sigma=\left(a_{i j}(\mathbf{x})\right), i, j=1, \ldots, d$, $\Psi(\mathbf{x}): \mathbb{R}^{d} \rightarrow \mathbb{R}^{d \times d}$ and $\mathbf{W}_{t} \in \mathbb{R}^{d}$, and $a_{i j}(\mathbf{x})=\sigma_{i}(\mathbf{x}) \sigma_{j}(\mathbf{x}) \rho_{i j}(\mathbf{x})$, where $\sigma_{i}(\mathbf{x})$ for all $i$ is the diffusion positive function for the $\mathrm{i}$-th process, and $\rho_{i j}(\mathbf{x}) \in[-1,1]$ is the pairwise correlation function between process $X_{t}^{i}$ and $X_{t}^{j}$.

In addition we specify an initial condition to Eq. A. 1 of the form $\mathbf{X}_{t}=x_{0}$, with $x_{0} \in$ $\mathbb{R}^{d}, t \geq 0$.

We can write the infinitesimal generator associated to Eq. A.1 as,

$$
A(x)=\sum_{i=1}^{d} b_{i}(x) \frac{\partial}{\partial x_{i}}+\frac{1}{2} \sum_{i, j=1}^{d} a_{i j}(x) \frac{\partial^{2}}{\partial x_{i} \partial x_{j}} .
$$

To understand this mathematically we recall the following results, we begin with some important notations. We denote a countably set by $\mathcal{X}^{k}:=\left\{x_{0}^{(k)}, \ldots, x_{n_{k}}^{(k)}\right\} \in \mathbb{R}, k=$ $1, \ldots, d$ as the state space for the Markov chain $X_{t}^{\left(n_{k}\right)}$ approximating $X_{t}^{k}$, with $X_{t}^{\left(n_{k}\right)} \rightarrow X_{t}^{k}$ in the weak sense as $n \rightarrow \infty$, being $n_{k}$ the number of discretization points for the kdimension state space. The continuous time Markov chain represents the mimicking BDP. We denote by $h_{k}=\left(x_{n_{k}}^{(k)}-x_{0}^{(k)}\right) / n_{k}$, the positive interval of the discretization grid $\mathcal{X}^{k}$.

The infinitesimal generator matrix entries, approximating the operator in Eq. A.2, are the local birth rate and death rate of the mimicking BDP and are calculated by matching the first and second instantaneous moments of the Markov chain $X_{t}^{\left(n_{k}\right)}$ that have to coincide with those of $X_{t}^{k}$ on the set $\mathcal{X}^{k, o}:=\mathcal{X}^{k} \backslash \partial \mathcal{X}^{k}$. This is achieved by local moment matching using the following equations,

$\mathbb{E}\left[\left(X_{t+\Delta t}^{k}-X_{t}^{k}\right)^{z} \mid X_{t}^{k}\right]=\mathbb{E}\left[\left(X_{t+\Delta t}^{\left(n_{k}\right)}-X_{t}^{\left(n_{k}\right)}\right)^{z} \mid X_{t}^{\left(n_{k}\right)}\right]+o(\Delta t), \quad z \in\{1,2\}$ and $X_{t}^{\left(n_{k}\right)} \in \mathcal{X}^{k}$

where the boundary $\partial \mathcal{X}$ consists of the smallest (i.e. $x_{0}^{(k)}$ ) and largest (i.e. $x_{n_{k}}^{(k)}$ ) elements in $\mathcal{X}^{k}$ and the interior $\mathcal{X}^{k, o}$ is the complement of the boundary.

In the following definition we illustrate the construction of the approximated infinitesimal generator matrix.

Definition 15 (Approximated Infinitesimal Generator matrix.) Let us denote by $A^{\left(n_{k}\right)}=$ $A_{X^{k}}^{\left(n_{k}\right)}=\left(a\left(x_{i}^{(k)}, x_{j}^{(k)}\right)\right)$ for $i, j=1, \ldots, n_{k}$ the approximated infinitesimal generator of the chain $X_{t}^{\left(n_{k}\right)}$ mimicking the Markov process $X_{t}^{k}$ with local drift parameters $b_{k}(\cdot)$ and diffusion parameter $\sigma_{k}(\cdot) . A^{\left(n_{k}\right)} \in \mathbb{R}^{n_{k} \times n_{k}}$ is the infinitesimal generator of the mimicking BDP $X_{t}^{\left(n_{k}\right)}$ and is a tridiagonal matrix, which entries are computed using Eq. A.3 yielding to:

$$
A_{X_{k}}^{\left(n_{k}\right)}=\left\{\begin{array}{l}
a\left(x_{0}^{(k)}, x_{1}^{(k)}\right)=a\left(x_{n_{k}}, x_{n_{k}-1}\right)=0, \\
a\left(x_{i}^{(k)}, x_{i+1}^{(k)}\right)=\frac{1}{2}\left(\frac{b_{k}\left(x_{i}^{(k)}\right)}{h_{k}}+\frac{\sigma_{k}^{2}\left(x_{i}^{(k)}\right)}{h_{k}^{2}}\right), \\
a\left(x_{i}^{(k)}, x_{i-1}^{(k)}\right)=\frac{1}{2}\left(\frac{\sigma_{k}^{2}\left(x_{i}^{(k)}\right)}{h_{k}^{2}}-\frac{b_{k}\left(x_{i}^{(k)}\right)}{h_{k}}\right), \\
a\left(x_{i}^{(k)}, x_{i}^{(k)}\right)=-\left(a\left(x_{i}^{(k)}, x_{i-1}^{(k)}\right)+a\left(x_{i}^{(k)}, x_{i+1}^{(k)}\right)\right),
\end{array}\right.
$$


with $-\frac{\sigma_{k}^{2}\left(x_{i}^{(k)}\right)}{h_{k}} \leq b_{k}\left(x_{i}\right) \leq \frac{\sigma_{k}^{2}\left(x_{i}\right)}{h}$ for all $k$, and $a\left(x_{i}, x_{j}\right) \geq 0$ for all $i, j$.

Remark A.1 The continuous drift and covariance functions introduced above are approximated as piecewise functions over the discretized support of mutidimensional CTMC, which means

$$
b_{k}(x)=b_{k}\left(x_{i}^{(k)}\right), \quad \sigma_{k}(x)=\sigma_{k}\left(x_{i}^{(k)}\right), \quad \text { if } x_{i}^{(k)} \leq x<x_{i+1}^{(k)}
$$

The link between the approximated terminal distribution $P_{t}^{\left(n_{k}\right)}$ of a Markov process at time $t$ and the infinitesimal generator matrix $A^{\left(n_{k}\right)}$ is given by the solution of the following Cauchy problem

$$
\frac{\partial P_{t}^{\left(n_{k}\right)}}{\partial t}=A^{\left(n_{k}\right)} P_{t}^{\left(n_{k}\right)}, \quad P_{0}^{\left(n_{k}\right)}=v .
$$

From a numerical point of view the calculation of $P_{t}^{\left(n_{k}\right)}$ in Eq. A.6 requires the computation of the matrix exponential $\exp \left(t A^{\left(n_{k}\right)}\right)$. One way to then tackle the curse of dimensionality that will arise when trying to evaluate such a matrix exponential in practice for large numbers of states, is to exploit the orthogonality across the Markov process dimensions.

In case of $d$ independent Markov processes, the infinitesimal generator

$$
A(x)=\sum_{i=1}^{d} b_{i}(x) \frac{\partial}{\partial x_{i}}+\frac{1}{2} \sum_{i=1}^{d} a_{i i}(x) \frac{\partial^{2}}{\partial x_{i}^{2}},
$$

is approximated by the following infinitesimal generator matrix:

$$
A^{(n)}=A_{X^{1}}^{\left(n_{1}\right)} \oplus A_{X^{2}}^{\left(n_{2}\right)} \oplus \ldots A_{X^{d}}^{\left(n_{d}\right)} \in \mathbb{R}^{n \times n}, \quad n=n_{1} n_{2} \cdots n_{d}
$$

where the symbol $\oplus$ denotes the Kroneker sum. We approximate (A.7) on a discrete multidimensional state space $\mathcal{X}=\bigoplus_{i=1}^{d} \mathcal{X}^{i}$, namely only over the orthogonal spaces. In this case the solution to Eq. A.6 is given by

$$
P_{t}^{(n)}=e^{t A^{\left(n_{1}\right)}} \otimes \cdots \otimes e^{t A^{\left(n_{d}\right)}}
$$

where the symbol $\otimes$ denotes the Kroneker product. We set the notation $P_{t}^{(n)}:=P_{t}^{(n) \perp}$, indicating the approximated density when the marginals are all orthogonal.

Remark A.2 Approximating over orthogonal dimension has a considerable numerical advantage. The problem of exponentiating the matrix $A^{(n)}$ becomes the exponential of single dimension (1-D) matrices which are the marginal generator matrices $A^{\left(n_{k}\right)}$, for all $k$.

We would like to exploit the approximation over orthogonal dimension also for the case of the generator in Eq. A.2, where there is a correlation structure across the Markov process' dimensions.

This is possible using a projected mimicking process constructed as a conditional BDP and which infinitesimal generator computation is proposed below.

Definition 16 (Conditional Local Infinitesimal Generator Matrix) Let $\mathbf{X}_{t}$ be a $d$ dimensional Markov process and $\mathbf{X}_{t}^{(n)}$ its corresponding approximating Markov chain. Let $X_{t}^{(n)}=\left(X_{t}^{\left(n_{k}\right)}, \mathbf{Y}_{t}^{\left(n^{\prime}\right)}\right)$ denote a partition of the chain, formed by the BDP process $X_{t}^{\left(n_{k}\right)}$, with $k \in(1, \ldots, d)$, and by d-1 BDP processes $\mathbf{Y}_{t}^{\left(n^{\prime}\right)}=\left(X_{t}^{\left(n_{j}\right)}\right)$ for $j=1, \ldots, d k \neq j$. Then, 
if we consider the conditional Markov chain $\left(X_{t}^{\left(n_{k}\right)} \mid \mathbf{Y}_{t}^{\left(n^{\prime}\right)}=\mathbf{y}\right)$, where $\mathbf{y} \in \mathcal{Y}:=\bigotimes_{\substack{i=1 \\ i \neq k}}^{d} \mathcal{X}^{k}$ is a state of the Markov chain partition $\mathbf{Y}_{t}^{\left(n^{\prime}\right)}$, we can calculate its infinitesimal generator matrix $A_{X_{k} \mid \mathbf{Y}}^{\left(n_{k}\right)}$ entries by matching the the first two instantaneous moments of $\left(X_{t}^{k} \mid \mathbf{Y}_{t}\right)$ and obtaining,

$$
A_{X_{k} \mid \mathbf{Y}}^{\left(n_{k}\right)}=\left\{\begin{array}{l}
a\left(x_{0}^{(k)}, x_{1}^{(k)}\right)=a\left(x_{n_{k}}, x_{n_{k}-1}\right)=0, \\
a\left(x_{i}^{(k)}, x_{i+1}^{(k)}\right)=\frac{1}{2}\left(\frac{b_{k}^{(c)}\left(x_{i}^{(k)}\right)}{h_{k}}+\frac{\sigma_{k}^{2,(c)}\left(x_{i}^{(k)}\right)}{h_{k}^{2}}\right), \\
a\left(x_{i}^{(k)}, x_{i-1}^{(k)}\right)=\frac{1}{2}\left(\frac{\sigma_{k}^{2,(c)}\left(x_{i}^{(k)}\right)}{h_{k}^{2}}-\frac{b_{k}^{(c)}\left(x_{i}^{(k)}\right)}{h_{k}}\right), \\
a\left(x_{i}^{(k)}, x_{i}^{(k)}\right)=-\left(a\left(x_{i}^{(k)}, x_{i-1}^{(k)}\right)+a\left(x_{i}^{(k)}, x_{i+1}^{(k)}\right)\right),
\end{array}\right.
$$

where the instantaneous conditional moments $\left(b_{k}^{(c)}, \sigma_{k}^{2,(c)}\right)$ are given by

$$
\left\{\begin{array}{l}
b_{k}^{(c)}\left(x_{i}^{(k)}\right)=b_{k}\left(x_{i}^{(k)}\right)+\Sigma_{y k} \Sigma_{k k}^{-1}\left(\mathbf{y}-b_{y}\right), \\
\sigma_{k}^{2,(c)}\left(x_{i}^{(k)}\right)=\Sigma_{y y}-\left(\Sigma_{y k} \Sigma_{k k}^{-1}\right) \Sigma_{k y}
\end{array}\right.
$$

for all $x_{i}^{(k)} \in \mathcal{X}^{k}$, and with moments partitions $\mathbf{b}(\mathbf{x})=\left[\begin{array}{l}b_{k} \\ b_{y}\end{array}\right]$ for the drift function and $\Sigma(\mathbf{x})=\left[\begin{array}{cc}\Sigma_{k k} & \Sigma_{k y} \\ \Sigma_{y k} & \Sigma_{y y}\end{array}\right]$ for the covariance matrix function respectively.

Example 1 (Two dimensional CTMC approximation) For example in a two dimensional case the conditional Markov chain $\left(X_{t}^{\left(n_{1}\right)} \mid X_{t}^{\left(n_{2}\right)}=x_{j}^{(2)}\right), x_{j}^{(2)} \in \mathcal{X}^{2}, j=1, \ldots, n_{2}$, has infinitesimal generator matrix

$$
A_{X_{1} \mid X_{2}=x_{j}^{(2)}}^{\left(n_{1}\right)}=\left\{\begin{array}{l}
a\left(x_{0}^{(1)}, x_{1}^{(1)}\right)=a\left(x_{n_{1}}, x_{n_{1}-1}\right)=0, \\
a\left(x_{i}^{(1)}, x_{i+1}^{(1)}\right)=\frac{1}{2}\left(\frac{b_{k}^{(c)}\left(x_{i}^{(1)}\right)}{h_{1}}+\frac{\sigma_{1}^{2,(c)}\left(x_{i}^{(1)}\right)}{h_{1}^{2}}\right), \\
a\left(x_{i}^{(1)}, x_{i-1}^{(k)}\right)=\frac{1}{2}\left(\frac{\sigma_{k}^{2,(c)}\left(x_{i}^{(1)}\right)}{h_{1}^{2}}-\frac{b_{1}^{(c)}\left(x_{i}^{(1)}\right)}{h_{1}}\right), \\
a\left(x_{i}^{(1)}, x_{i}^{(1)}\right)=-\left(a\left(x_{i}^{(1)}, x_{i-1}^{(1)}\right)+a\left(x_{i}^{(1)}, x_{i+1}^{(1)}\right)\right),
\end{array} \quad \text { for all } j,\right.
$$

where the instantaneous conditional moments $\left(b_{k}^{(c)}, \sigma_{k}^{2,(c)}\right)$ are given by

$$
\left\{\begin{array}{l}
b_{1}^{(c)}\left(x_{i}^{(1)}\right)=b_{1}\left(x_{i}^{(1)}\right)+\rho\left(x_{i}^{(1)}, x_{j}^{(2)}\right) \frac{\sigma_{1}\left(x_{i}^{(1)}\right)}{\sigma_{2}\left(x_{j}^{(2)}\right)}\left(x_{j}^{(2)}-b_{2}\right), \\
\sigma_{1}^{2,(c)}\left(x_{i}^{(1)}\right)=\sigma_{1}^{2}\left(x_{i}^{(1)}\right)\left(1-\rho^{2}\left(x_{i}^{(1)}, x_{j}^{(2)}\right)\right)
\end{array} \quad \text { for all } j .\right.
$$

Then one of the useful results derived in Dalessandro and Peters (2016) which we will utilise in this paper is the following approximation of the matrix exponential of the Kronecker sum of a sequence of matrices,

$$
\exp \left(A \oplus_{S}\left\{B_{j}\right\}\right) \approx \exp (A) \otimes_{S} \exp \left(\left\{B_{j}\right\}\right)+O\left(\left[A,\left\{B_{j}\right\}\right]\right) .
$$

where the notation $\left\{B_{j}\right\}, j=1,2, \ldots$ denotes a sequence of matrices $\left\{B_{1}, B_{2}, \ldots\right\}$. In Eq. A.14 we utilise the definition that if $A$ is an $\mathbb{R}^{m \times m}$ matrix and $\left\{B_{j}\right\}, j=1, \ldots, m$ is a 
sequence of $m$ matrices in $\mathbb{R}^{n \times n}$, then the Kronecker product of a matrix sequence $A \otimes_{S}\left\{B_{j}\right\}$ is the $n m \times n m$ block matrix:

$$
A \otimes_{S}\left\{B_{j}\right\}=\left[\begin{array}{lll}
a_{11} B_{1} & \cdots & a_{1 m} B_{m} \\
\vdots & \ddots & \vdots \\
a_{m 1} B_{1} & \cdots & a_{m m} B_{m}
\end{array}\right] .
$$

In general, the construction of the infinitesimal generator matrix by means of the conditional operators is done using the following tensor equation:

$$
A_{X}^{(n)}=A_{X_{1}}^{\left(n_{1}\right)} \oplus_{S}\left\{A_{X_{2} \mid X_{1}}^{\left(n_{2}\right)}\right\} \oplus_{S} \cdots \oplus_{S}\left\{A_{X_{d} \mid X_{1}, \ldots, X_{d-1}}^{\left(n_{1}\right)}\right\}
$$

where $\oplus_{S}$ denotes the Kronecker sum over a matrix sequence.

In this way, using the approximation in Eq. A.14, it is possible to compute the transition density for the multidimensional Markov chain, which is given by

$$
P_{t}^{(n)}=e^{t A_{X_{1}}^{\left(n_{1}\right)}} \otimes_{S}\left\{e^{t A_{X_{2} \mid X_{1}}^{\left(n_{2}\right)}}\right\} \cdots \otimes_{S}\left\{e^{t A_{X_{d} \mid X_{1}, \ldots, X_{d-1}}^{\left(n_{d}\right)}}\right\}
$$

\section{Convergence Discussion for Discrete Approximation}

For completeness we report some convergence results of the proposed approximation scheme from Dalessandro and Peters (2017), which are in its Section 4 entitled 'Convergence of the approximated Generator'. In our methodology we construct a continuous Markov chain $X^{(n)}$ with dynamics as close as possible to the corresponding approximated process $\left(X_{t}\right)_{t \geq 0}$. At this purpose we define and study the following error

$$
\varepsilon_{n}(f)=\sup _{x \in \mathcal{X}^{o}}\left\|A^{(n)} f(x)-A f(x)\right\|_{2}=c O\left((\Delta x)^{2}\right) \stackrel{n \rightarrow \infty}{\longrightarrow} 0
$$

which, according to Theorem 13 in Dalessandro and Peters (2017) implies that also the Markov chain transition density at time $t$ converges to the process transition density at the same time, namely $P_{t}^{(n)} \pi_{n} f \rightarrow P_{t} f$, for all $f$ in the core of $A$, where $\pi_{n}$ is an auxiliary mapping function. The theoretical result of convergence states that the error is at most some constant $c$ times $\left|(\Delta x)^{2}\right|$ when $\Delta x$ is close to zero.

We illustrate this theoretical result in Fig. 17, where we consider a $d=5$ dimensional diffusion with drift and diffusion functions equal to $b_{k}(x)=0, \sigma_{k}(x)=1$ for all $k$ respectively and we closely study the cases of correlation $\rho(x)=0$ and $\rho(x) \neq 0$.

The plots report the error function $\epsilon(n)=\left\|P_{t}-P_{t}^{(n)}\right\|_{2}$, which is the $L^{2}$-norm of the difference between the Markov chain transition density at time $t$ and the original process transition density at the same time, as the number of states in each dimension $n_{k}=n$ for all $k$, goes towards infinity.

The empirical study of the scheme convergence meets and satisfies the theoretical findings. In particular we can observe the consistent agreement of the schemes convergence rate when correlation is zero. We can furthermore notice how non-zero correlation $(\rho(x)= \pm 0.7)$ makes convergence lower.

In all the examples and empirical studies and in the concordance measure analysis we use $n=200$ grid points across all chain dimensions when constructing an approximating Markov chain. This is a number of grid points which guarantees a convergence error $\leq 10^{-4}$ according to Fig. 17. 


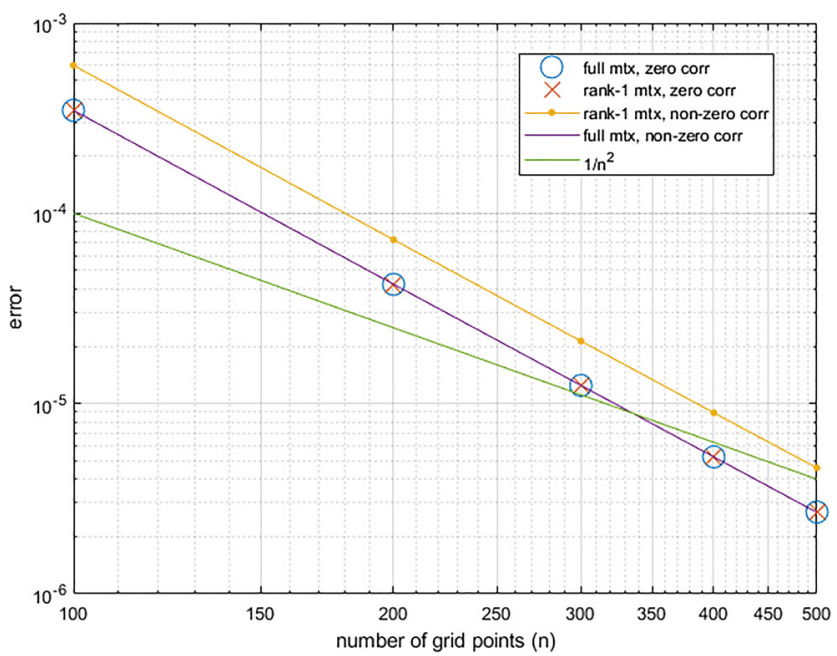

Fig. 17 The plot reports the convergence rate error of the proposed CTMC approximation scheme, as a function of $n$, the number of grid points in each dimension. The chain approximates a $d=5$ with drift and diffusion functions equal to $b_{k}(x)=0, \sigma_{k}(x)=1$ for all $k$ respectively. The cases of correlation $\rho(\mathbf{x})=0$ and $\rho(\mathbf{x}) \neq 0,(\rho(\mathbf{x})= \pm 0.7)$ are reported. There is consistent agreement of the schemes convergence rate when correlation is zero. In fact, the blue circles display the convergence of a full generator matrix with zero correlations. Identical convergence rates are displayed for the full generator matrix with non-zero correlation and the rank-1 generator matrix with zero correlation. The rate of convergence is lower for the rank-1 generator matrix with non-zero correlation equal to $(\rho(\mathbf{x})= \pm 0.7)$. Note that the convergence error functions are benchmarked against the function $1 / n^{2}$, which is the reference rate for the theoretical results

\section{Appendix B: Construction of the Mimicking Local Gaussian Copula}

This appendix provides a practical summary of the key results and algorithms from Dalessandro and Peters (2016) about the approximated local Gaussian copula construction, which include:

1. Construction of the approximated local Gaussian copula density $c^{(n)}$ as the ratio of a joint terminal distribution $P_{t}^{(n)}$ and the corresponding independent terminal distribution $P_{t}^{(n) \perp}$, see Eq. 1.7. This is done within the discretized hypercube $\mathbf{U}=\bigotimes_{k=1}^{d} \mathbf{U}^{k}$ support of the multidimensional CTMC.

2. Mapping of the approximated local Gaussian copula $C^{(n)}$ into any target copula distributions evaluated pointwise on the hypercube $\mathbf{U}$.

The construction of the mimicking local Gaussian copula function $c^{(n)}$ can be obtained as a decomposition of the generator matrix into the approximated operator matrix of a generic copula function and the marginal approximated densities' operators Kronecker product. This important result from Dalessandro and Peters (2016), which we report below, clearly show how the approximated local Gaussian copula density $c^{(n)}$ is the ratio of a joint terminal distribution $P_{t}^{(n)}$ and the corresponding independent terminal distribution $P_{t}^{(n) \perp}$.

Proposition (CTMC Functional Copula Infinitesimal Operator) Let $\mathbf{X}_{t}^{(n)}:=\left(X_{t}^{\left(n_{1}\right)}, X_{t}^{\left(n_{2}\right)}\right)$ be a bivariate continuous time Markov chain with approximate weak solution $P_{t}^{(n)}$, 
mimicking the correlated diffusion $\mathbf{X}_{t}:=\left(X_{t}^{1}, X_{t}^{2}\right)$. Then the CTMC mimicking process can be characterized by the bivariate functional copula density operator representation given by

$$
c^{(n)}=c^{\left(n_{1} n_{2}\right)}:=I^{\left(n_{1}\right)} \otimes_{S}\left\{\exp \left(t \hat{A}_{X^{2} \mid X^{1}}^{\left(n_{2}\right)}\right)\right\}
$$

where $I^{\left(n_{1}\right)} \in \mathbb{R}^{n_{1} \times n_{1}}$ is the unitary matrix, and $\left\{\hat{A}_{X^{2} \mid X^{1}}^{\left(n_{2}\right)}\right\}$ is the operator matrix sequence of the linear least square estimator of the conditional Markov chain process $\left(X_{t}^{\left(n_{1}\right)} \mid X_{t}^{\left(n_{2}\right)}\right)$.

Proof In order to calculate the approximated expression for the mimicking copula density $c^{(n)}$, we first look at how we can rewrite the approximate solution $P^{(n)}$. We have:

$$
\begin{aligned}
P_{t}^{(n)}:=P_{X_{t}^{1}, X_{t}^{2}}^{\left(n_{1} n_{2}\right)} & \approx P_{X_{t}^{1}}^{\left(n_{1}\right)} \otimes_{S}\left\{P_{X_{t}^{2} \mid X_{t}^{1}}^{\left(n_{2}\right)}\right. \\
& =\exp \left(t A_{X^{1}}^{\left(n_{1}\right)}\right) \otimes_{S}\left(\left\{\exp \left(t A_{X^{2} \mid X^{1}}^{\left(n_{2}\right)}\right)\right\}\right) \\
& =\exp \left(t A_{X^{1}}^{\left(n_{1}\right)}\right) \otimes_{S}\left(\exp \left(t A_{X^{2}}^{\left(n_{2}\right)}\right) \times\left\{\exp \left(t \hat{A}_{X^{2} \mid X^{1}}^{\left(n_{2}\right)}\right)\right\}\right) \\
& =\left(\exp \left(t A_{X^{1}}^{\left(n_{1}\right)}\right) \otimes \exp \left(t A_{X^{2}}^{\left(n_{2}\right)}\right)\right) \times\left(I^{\left(n_{1}\right)} \otimes_{S}\left\{\exp \left(t \hat{A}_{X^{2} \mid X^{1}}^{\left(n_{2}\right)}\right)\right\}\right)
\end{aligned}
$$

Then we can rewrite

$$
c^{(n)}=I^{\left(n_{1}\right)} \otimes_{S}\left\{\exp \left(t \hat{A}_{X^{2} \mid X^{1}}^{\left(n_{2}\right)}\right)\right\}=\frac{P_{t}^{(n)}}{\exp \left(t A_{X^{1}}^{\left(n_{1}\right)}\right) \otimes \exp \left(t A_{X^{2}}^{\left(n_{2}\right)}\right)}=\frac{P_{t}^{(n)}}{P_{t}^{(n) \perp}}
$$

The copula operator in the d-dimensional case was shown to be of the form:

$$
I^{\left(n_{1}\right)} \otimes_{S}\left\{\exp \left(A_{X_{2} \mid X_{1}}^{\left(c, n_{2}\right)}\right)\right\} \otimes_{S} \cdots \otimes_{S}\left\{\exp \left(A_{X_{d} \mid X_{d-1}, \ldots, X_{1}}^{\left(c, n_{d}\right)}\right)\right\}
$$

In Section 3 of Dalessandro and Peters (2016) they then show how to utilise this decomposition of the generalized diffusions generator to locally reconstruct what they term a generalized Gaussian copula reconstruction of any target copula model. In fact this is possible through the mapping

$$
\min _{\rho(\mathbf{u})}\left\|C_{\text {target }}^{(n)}\left(\mathbf{u} ; \theta_{c}\right)-C^{(n)}(\mathbf{u} ;\{\theta, \rho(\mathbf{u})\})\right\|_{2}
$$

for all $\mathbf{u} \in \mathbf{U}$, where

$$
C^{(n)}\left(B_{z}\right)=\sum_{j \leq z} c^{(n)}\left(B_{j}\right)
$$

where the support $\mathcal{X}$ is represented as union of disjoint subsets $\left\{B_{j}\right\}$, i.e. $\mathcal{X}=\bigotimes_{i=1}^{d} \mathcal{X}^{i}=$ $\bigcup_{j=1}^{n} B_{j}$, with each set $B_{j}$ being a coordinate vector point $\mathbf{x}$ in the space $\mathcal{X}$ and $B_{0}=\{\mathbf{x}=$ $\left.\left(x_{0}^{(1)}, \ldots, x_{0}^{(d)}\right)\right\}, B_{1}=\left\{\mathbf{x}=\left(x_{1}^{(1)}, \ldots, x_{1}^{(d)}\right)\right\}, \ldots, B_{n}=\left\{\mathbf{x}=\left(x_{n_{1}}^{(1)}, \ldots, x_{n_{d}}^{(d)}\right)\right\}$ being a countable and ordered sequence of sets spanning the whole discretized support $\mathcal{X}$.

\section{References}

Blomqvist N (1950) On a measure of dependence between two random variables. Ann Math Stat, 593-600 Cambanis S, Simons G, Stout W (1976) Inequalities for $E_{k}(x, y)$ when the marginals are fixed. Zeitschrift für Wahrscheinlichkeitstheorie und verwandte Gebiete 36(4):285-294 
Cruz MG, Peters GW, Shevchenko PV (2015) Fundamental aspects of operational risk and insurance analytics: a handbook of operational risk. Wiley, Handbook of Financial Engineering and Econometrics

Dalessandro A, Peters GW (2016) Tensor approximation of generalized correlated diffusions for decomposing copulas: part A. Available at SSRN: https://ssrn.com/abstract=2813135

Dalessandro A, Peters GW (2017) Tensor approximation of generalized correlated diffusions and functional copula operators. Methodol Comput Appl Probab, 1-35

De Luca G, Rivieccio G (2012) Multivariate tail dependence coefficients for Archimedean copulae. Pages 287-296 of: advanced statistical methods for the analysis of large data-sets. Springer

Dolati A, Úbeda-Flores M (2006) On measures of multivariate concordance. J Probab Stat Sci 4(2):147-164

Embrechts P, Lindskog F, McNeil A (2001) Modelling dependence with copulas. Rapport technique Département de mathématiques, publisher=Institut Fédéral de Technologie de Zurich, address=Zurich

Fredricks GA, Nelsen RB (2007) On the relationship between Spearman's rho and Kendall's tau for pairs of continuous random variables. J Stat Planning Inference 137(7):2143-2150

Galton F (1894) Natural inheritance. Macmillan

Joe H (1990) Multivariate concordance. J Multivar Anal 35(1):12-30

Joe H (1997) Multivariate models and multivariate dependence concepts. CRC Press

Joe H, Li H, Nikoloulopoulos AK (2010) Tail dependence functions and vine copulas. J Multivar Anal 101(1):252-270

Kendall MG (1938) A new measure of rank correlation. Biometrika, 81-93

Klüppelberg C, Kuhn G, Peng L (2008) Semi-Parametric models for the multivariate tail dependence function-the asymptotically dependent case. Scand J Stat 35(4):701-718

Li H (2009) Orthant tail dependence of multivariate extreme value distributions. J Multivar Anal 100(1):243256

Mari DD, Kotz S (2001) Correlation and dependence, vol. 518. World Scientific

Nelsen RB (1999) An introduction to copulas, vol 139. Springer Science \& Business Media

Nelsen RB (2002) Concordance and copulas: a survey. In: Cuadras CM, Fortiana J, Rodriguez-Lallena JA (eds) Pages 169-177 of: distributions with given marginals and statistical modelling. Springer, Dordrecht

Pearson K (1896) Mathematical contributions to the theory of evolution.-On a form of spurious correlation which may arise when indices are used in the measurement of organs. Proc R Soc Lond 60(359-367):489-498

Scarsini M (1984) On measures of concordance. Stochastica: revista de matemática pura y aplicada 8(3):201218

Spearman C (1904) "General intelligence," objectively determined and measured. Am J Psychol 15(2):201292

Sungur EA (2005) A note on directional dependence in regression setting. Commun Stat - Theory Methods 34(9-10):1957-1965

Taylor MD (2007) Multivariate measures of concordance. Ann Inst Stat Math 59(4):789-806

Tchen AH (1980) Inequalities for distributions with given marginals. Annals Probab, 814-827

Yanagimoto T, Okamoto M (1969) Partial orderings of permutations and monotonicity of a rank correlation statistic. Ann Instit Stat Math 21(1):489-506

Publisher's Note Springer Nature remains neutral with regard to jurisdictional claims in published maps and institutional affiliations. 